\title{
Parental and/or Personal Socioeconomic Circumstances: How to Explain Site-Specific Cancer Mortality Inequalities Among Young Belgian Adults
}

\author{
Katrien Vanthomme*, Hadewijch Vandenheede, Paulien Hagedoorn and Sylvie Gadeyne \\ Vrije Universiteit Brussel, Department of Sociology, Interface Demography, Pleinlaan 2, 1050 Brussels, Belgium \\ "Corresponding Author: Dr. Katrien Vanthomme, Vrije Universiteit Brussel, Department of Sociology, \\ Interface Demography, Pleinlaan 2, 1050 Brussels, Belgium, Tel: +32 261481 28; Fax: +32 261481 35; E-mail: \\ Katrien.Vanthomme@vub.ac.be
}

Received: 19 March 2018; Accepted: 06 April 2018; Published: 13 April 2018

\begin{abstract}
Both socioeconomic position (SEP) in childhood and adulthood are associated with adult mortality. Yet, the relative importance of childhood socioeconomic (SE) conditions is likely to vary depending on the cause of death. This paper will study: 1) whether SE conditions during both childhood and adulthood are associated with overall and sitespecific cancer mortality among Belgian young adults; 2) the relative importance of all indicators of parental and personal SEP in explaining differences in cancer mortality.
\end{abstract}

Data were derived from record linkage between the Belgian censuses of 1991 and 2001 and mortality follow-up data for 2001-2011. Parental educational attainment, parental occupational status, parental housing status, and childhood area deprivation were used as measures of parental SEP, and current education as indicator of personal SEP. SE differences were studied for overall and site-specific cancer mortality by calculating age-standardized mortality rates and mortality rate ratios using Poisson regression.

Both low parental and personal SEP are associated with higher all-cancer mortality in young adulthood. Personal education seemed to be the strongest indicator of overall and site-specific cancer mortality in young adulthood, independent of parental SEP. Yet, for women, this was partly due to a health selection effect. In the full models, parental SEP was no longer associated with cancer mortality but deprived living conditions still were. Site-specific inequalities were generally more expressed among women than among men. Studies and policies focussing on SE inequalities in mortality in young adulthood should allow for both childhood and adulthood SE conditions. 
Keywords: Socioeconomic position; Cancer; Mortality; Inequalities; Belgium; Young adults; Life-course perspective

\section{Introduction}

Ample studies have shown that not only socioeconomic position (SEP) in adulthood, but also adverse early life circumstances are associated with increased adult mortality [1-4]. Yet, the relative importance of childhood social conditions may vary depending on the cause of death (COD) [5-7]. For some COD, such as cardiovascular diseases, the association with social conditions in childhood is evident, whereas for cancer mortality, evidence is inconsistent $[1,3]$. Also, little is known about whether this association is similar for men and women [8,9]. This pattern could be different because of gender differences in the socioeconomic (SE) distribution of risk factors, e.g. health-related behaviours [8,9]. According to the life-course perspective, the social patterning of cause-specific adult mortality is associated with SE inequalities in exposures at different life stages [2, 10-13]. These exposures may act during critical periods in childhood, adolescence and adulthood, and may have long-lasting effects on health [3,11, 12, 14]. Moreover, adverse effects of poor SE conditions across different life stages may accumulate over the life course, resulting in social inequalities in health $[4,11,14]$.

Mapping out the relation between early life SE conditions and cause-specific mortality in (young) adulthood can provide insights in disease aetiology. This is important to develop interventions that prevent and reduce SE inequalities in premature mortality [1, 3, 8, 15]. De Grande et al. [16] already mapped out the social distribution of young adult mortality for the five most common COD in Flanders and Brussels in 2001-2009, using a life-course perspective. We will elaborate this knowledge by studying the social distribution of site-specific cancer mortality in Belgian young adults.

We will add to the literature by addressing three research questions (RQ). First, are parental and personal SEP associated with young adult (site-specific) cancer mortality? Secondly, what is the relative importance of parental and personal SEP indicators in explaining cancer mortality differences: can the association between young adult cancer mortality and parental SEP be attenuated by personal SEP achieved during early adulthood? Additionally, we will assess whether the observed patterns are similar for men and women. While most studies use only one indicator of parental SEP (often parental occupation) [16], we will include four indicators of parental SEP: parental education, occupation, housing conditions, and area deprivation.

\section{Material and Methods}

\subsection{Design and study population}

Data were derived from record linkage between the Belgian censuses of 1991 and 2001 and register data on emigration and mortality for the follow-up period 01/10/2001-31/12/2011. This unique source of information contains information on mortality, emigration, COD, sociodemographic (SD) and SE variables of all individuals legally residing in Belgium at the time of the censuses. 
All Belgian inhabitants aged 8-18 at the census of 1991, who lived in a private household together with (at least one of) the parents and who were still alive at the 2001 census were included. By linking these children to their parents in the census of 1991, we were able to derive the social circumstances during childhood. As we followed these individuals up to the 2001 census, we could determine their personal SEP during young adulthood as well. Those with missing information on parental or personal SEP were excluded from the analyses (15.01\%).

\subsection{Variables}

We measured SEP at two instants: during childhood (census 1991) and young adulthood (census 2001). As indicators of childhood SEP, parental educational attainment, parental occupational status, housing status were used, as well as the area deprivation of the municipality the household lived in. Parental education was operationalized as the highest obtained degree by one of the parents and consists of three categories: lower secondary education or less (ISCED 0-2, "low"), higher secondary (ISCED 3-4, “mid"), and tertiary education (ISCED 5-6, "high"). Parental occupational status was operationalized by taking the occupational status of the father, and in case of single-mum households the occupational status of the mother. This variable consists of three categories: employed, looking for a job while receiving an unemployment allowance, and unemployed. This latter category includes people that are retired $(60 \%)$, not working and not looking for a job (22\%), disabled (13\%) and housekeepers $(5 \%)$. The indicator housing conditions consists of a combination of ownership (tenant or owner) and comfort level of the house (low-, mid- and high-comfort dwellings), resulting in six categories [17]. The area deprivation of the municipality of residence during childhood was used as an additional indicator of childhood SEP. This deprivation index is based on the Carstairs index, adapted to the Belgian context [18]. The index is based on three variables: the percentage unemployed men aged 18-64, the percentage households without a car, and the percentage of low-educated inhabitants aged 25-64 years within each municipality. The index is constructed by taking the sum of the z-scores of these three variables by municipality, weighted by the population size of the municipality at the census [19]. The municipalities are ranked on their score and grouped into quintiles of equal sizes, ranging from the least deprived (Q1) to the most deprived municipalities (Q5). Using the linked census data of 2001, we were able to use current education as indicator of personal SEP as well. Since the study population is aged 19-29 years at the 2001 census, most individuals should have finalized secondary education, making it possible to distinguish lower from higher educated young adults. Personal education was measured by the highest educational degree if not enrolled in education at the census. For those currently enrolled in education, the educational level currently enrolled in was used. This allows us to make a distinction between lower secondary education or less ("low"), higher secondary education ("mid") and higher education ("high"). We decided not to include area deprivation, nor housing conditions during adulthood as indicators of personal SEP because the majority (58\%) of young adults still lives with their parents at the 2001 census. Moreover, we did not adjust for personal occupational status because one quarter of the study population was still full-time enrolled in education at the 2001 census.

Deaths were followed over the period 01/10/2001 to 31/12/2011. Age was included as a time-varying covariate to account for age changes during this 11-year follow-up. To do so, individual follow-up time was split into episodes consisting of 1-year attained age groups using Lexis expansions, which allows a follow-up period for mortality from 
19 years to a maximum of 39 years. SE differences were studied for all-cancer mortality and for the most common cancer mortality sites within this study population, representing at least five percent of total cancer mortality $(\mathrm{N} \geq 25)$. For the cancer sites, corresponding ICD-10 codes and number of deaths, see Table 1 .

\begin{tabular}{|l|l|l|l|l|l|}
\hline \multirow{2}{*}{ Cancer site } & \multirow{2}{*}{ ICD-10 } & Men & \multicolumn{2}{l|}{ Women } \\
\cline { 3 - 6 } & & N & \% & N & \% \\
\hline Colorectum & C18-21 & 25 & 4.9 & 31 & 5 \\
\hline Lung & C33-34 & 35 & 6.8 & - & - \\
\hline Bone and articular cartilage & C40-41 & 35 & 6.8 & - & - \\
\hline Malignant melanoma & C43 & 54 & 10.5 & 38 & 6.1 \\
\hline Soft tissue & C46-49 & - & - & 34 & 5.5 \\
\hline Breast & C50 & - & - & 140 & 22.6 \\
\hline Cervix uteri & C53 & - & - & 28 & 4.5 \\
\hline Ovary & C56 & - & - & 29 & 4.7 \\
\hline Brain & C71 & 87 & 16.9 & 68 & 11 \\
\hline Non-Hodgkin lymphoma & C82-85 & 34 & 6.6 & 29 & 4.7 \\
\hline Leukaemia & C91-95 & 73 & 14.2 & 65 & 10.5 \\
\hline All cancers & C00-97 & 515 & 66.6 & 619 & 74.6 \\
\hline
\end{tabular}

Table 1: Distribution of cancer deaths among Belgian young adults, 2001-2011.

\subsection{Statistical analyses}

To answer the first RQ, age-standardized mortality rates (ASMR) for overall and site-specific cancer mortality were calculated for all indicators of parental and SEP. These ASMRs are directly standardized to the total Belgian population and stratified by sex. Moreover, relative measures were calculated by means of Poisson regression. Ageadjusted mortality rate ratios (MRR) have been calculated for all SEP indicators, and for each COD. To answer the second and third RQ, age-adjusted multilevel Poisson models were calculated for overall and site-specific cancers, including all parental and personal SEP indicators. As parental area deprivation is measured at the municipality level, these analyses were done by means of multilevel Poisson models, using the log of the person years as an offset. To exclude a health selection effect regarding the association between personal education and cancer mortality, we performed some sensitivity analyses adjusting for self-rated health (SRH) at the 2001 census (ranging from very poor to very good). These results are not shown but discussed when relevant (see results and discussion). All analyses were stratified by sex and performed using STATA 13.1.

\section{Results}

\subsection{Description of the study population}

The study population consists of about 1.3 million Belgian young adults of which 515 men and 619 women died from cancer during the follow-up period 2001-2011 (Table 2). This results in an ASMR of 9.5/100,000 women (95\% C.I. 8.8-10.3), and 7.6/100,000 men (95\% C.I. 6.9-8.3). For more than half of the young adults, highest parental educational level is at least upper secondary education, and for one quarter even tertiary education. In 
comparison, personal education is much higher: $36 \%$ of the men and $49 \%$ of the women included in the study population attained (or are enrolled) in tertiary education. About $15 \%$ of the young adults grew up in a family where the father was not working at the time of the 1991 census, either unemployed or looking for a job. About one quarter grew up in a rented house, and about one third lived in poor housing conditions.

\begin{tabular}{|c|c|c|c|c|c|c|c|c|c|c|}
\hline \multicolumn{6}{|l|}{ Men } & \multicolumn{5}{|c|}{ Women } \\
\hline & \multirow{3}{*}{$\begin{array}{l}\mathbf{N} \\
672,605\end{array}$} & \multirow{3}{*}{$\%$} & \multirow{3}{*}{$\begin{array}{l}\text { Cancer } \\
\text { deaths } \\
515 \\
\end{array}$} & \multicolumn{2}{|c|}{ ASMR } & \multirow{3}{*}{$\begin{array}{l}\mathbf{N} \\
642,497\end{array}$} & \multirow[t]{3}{*}{$\%$} & \multirow{3}{*}{$\begin{array}{l}\text { Cancer } \\
\text { deaths } \\
619\end{array}$} & \multirow{2}{*}{\multicolumn{2}{|c|}{\begin{tabular}{|c|} 
ASMR \\
(95\% C.I. $)$
\end{tabular}}} \\
\hline & & & & \multicolumn{2}{|c|}{ (95\% C.I.) } & & & & & \\
\hline \multirow{2}{*}{\multicolumn{11}{|c|}{\begin{tabular}{l|l}
$(6.9-8.3)$ & 642,497 \\
\end{tabular}}} \\
\hline & & & & & & & & & & \\
\hline \begin{tabular}{l|l} 
Parental education \\
Low & 284,542
\end{tabular} & 284,542 & 42.3 & 244 & 8.3 & $(7.2-9.3)$ & 272,241 & 42.4 & 287 & 10 & $(8.9-11.2)$ \\
\hline Mid & 175,213 & 26.1 & 142 & 8.3 & $(6.9-9.7)$ & 167,284 & 26 & 164 & 10.1 & $(8.6-11.7)$ \\
\hline High & 172,686 & 25.7 & 101 & 6 & $(4.8-7.1)$ & 164,311 & 25.6 & 123 & 7.7 & $(6.3-9.0)$ \\
\hline Missing & 40,164 & 6 & 28 & 7 & $(4.4-9.6)$ & 38,661 & 6 & 45 & 11.4 & $(8.1-14.8)$ \\
\hline \multicolumn{11}{|c|}{ Parental occupation } \\
\hline Employed & 552,877 & 82.2 & 425 & 7.7 & $(7.0-8.4)$ & 526,973 & 82 & 494 & 9.4 & $(8.6-10.2)$ \\
\hline $\begin{array}{l}\text { Looking for a } \\
\text { job }\end{array}$ & 43,198 & 6.4 & 30 & 7 & $(4.5-9.6)$ & 41,765 & 6.5 & 38 & 9.2 & $(6.2-12.1)$ \\
\hline Unemployed & 58,398 & 8.7 & 44 & 6.7 & $(4.5-8.8)$ & 56,277 & 8.8 & 69 & 10.3 & $(7.8-12.9)$ \\
\hline Missing & 18,132 & 2.7 & 16 & 8.7 & $(4.4-13.0)$ & 17,482 & 2.7 & 18 & 10.1 & $(5.4-14.8)$ \\
\hline \multicolumn{11}{|c|}{ Parental housing conditions } \\
\hline Low tenant & 74,056 & 11 & 66 & 9.1 & $(6.9-11.3)$ & 70,607 & 11 & 85 & 12 & $(9.5-14.6)$ \\
\hline Mid tenant & 30,025 & 4.5 & 28 & 9.4 & $(5.9-12.9)$ & 29,531 & 4.6 & 23 & 7.9 & $(4.7-11.1)$ \\
\hline High tenant & 56,956 & 8.5 & 39 & 7 & $(4.8-9.2)$ & 55,172 & 8.6 & 39 & 7.1 & $(4.9-9.4)$ \\
\hline Low owner & 149,338 & 22.2 & 126 & 8.3 & $(6.9-9.8)$ & 141,802 & 22.1 & 163 & 11.4 & $(9.7-13.2)$ \\
\hline Mid owner & 46,686 & 6.9 & 33 & 7 & $(4.6-9.3)$ & 44,438 & 6.9 & 38 & 8.1 & $(5.5-10.7)$ \\
\hline High owner & 291,806 & 43.4 & 204 & 6.9 & $(6.0-7.9)$ & 277,835 & 43.2 & 247 & 8.7 & $(7.6-9.8)$ \\
\hline Missing & 23,738 & 3.5 & 19 & 8 & $(4.4-11.6)$ & 23,112 & 3.6 & 24 & 10.4 & $(6.2-14.6)$ \\
\hline \multicolumn{11}{|c|}{ Parental area deprivation } \\
\hline Q1 & 122,882 & 18.3 & 97 & 7.9 & $(6.3-9.4)$ & 118,712 & 18.5 & 112 & 9.4 & $(7.7-11.1)$ \\
\hline Q2 & 115,543 & 17.2 & 100 & 8.6 & $(6.9-10.3)$ & 110,654 & 17.2 & 121 & 10.8 & $(8.9-12.7)$ \\
\hline Q3 & 122,036 & 18.1 & 99 & 8 & $(6.4-9.6)$ & 116,439 & 18.1 & 108 & 9.2 & $(7.4-10.9)$ \\
\hline $\mathrm{Q} 4$ & 127,528 & 19 & 98 & 7.6 & $(6.1-9.1)$ & 121,970 & 19 & 120 & 9.7 & $(8.0-11.5)$ \\
\hline Q5 & 138,223 & 20.6 & 87 & 6.3 & $(4.9-7.6)$ & 131,857 & 20.5 & 115 & 8.7 & $(7.1-10.3)$ \\
\hline Missing & 46,393 & 6.9 & 34 & 7.4 & $(4.9-9.9)$ & 42,865 & 6.7 & 43 & 9.9 & $(7.0-12.9)$ \\
\hline \multicolumn{11}{|c|}{ Personal education } \\
\hline Low & 114,051 & 17 & 131 & 11.3 & $(9.3-13.2)$ & 69,868 & 10.9 & 79 & 10.9 & $(8.5-13.3)$ \\
\hline Mid & 283,422 & 42.1 & 207 & 7.3 & $(6.3-8.3)$ & 237,299 & 36.9 & 265 & 11.1 & $(9.8-12.4)$ \\
\hline High & 239,333 & 35.6 & 144 & 5.9 & $(5.0-6.9)$ & 310,750 & 48.4 & 233 & 7.4 & $(6.5-8.4)$ \\
\hline Missing & 35,799 & 5.3 & 33 & 9.6 & $(6.3-12.9)$ & 24,580 & 3.8 & 42 & 17.7 & $(12.3-23.0)$ \\
\hline
\end{tabular}

Table 2: Description of childhood and adult socioeconomic circumstances among Belgian young adults. 
3.2 Are parental and personal SEP associated with cancer mortality during young adulthood?

All SEP indicators are inversely associated with overall cancer mortality, both in men and women, except for parental occupation (Tables 2 and 3, model 1). Low parental education results in a 38\% higher all-cancer mortality rate (MR) (95\% C.I. 1.09-1.74) in young adulthood for men. Housing conditions during childhood are also related to adult cancer mortality. Living in a house of poor quality results in higher overall cancer mortality. Furthermore, the deprivation of the area where they were raised shows mortality inequalities, with higher overall cancer mortality among young adult men growing up in the deprived areas (MRR Q4: 1.36, 95\% C.I. 1.02-1.82). Personal SEP also matters: low-educated young adults have a MRR of 1.91 (95\% C.I. 1.51-2.43) in men and 1.48 (95\% C.I. 1.15-1.91) in women compared with the high-educated. Sensitivity tests (results not shown) adjusting for SRH, show that for men the association between personal education and cancer mortality remains, while in women the association is explained for low-educated women. This could point to a health selection effect: these women were already too ill to finish their education.

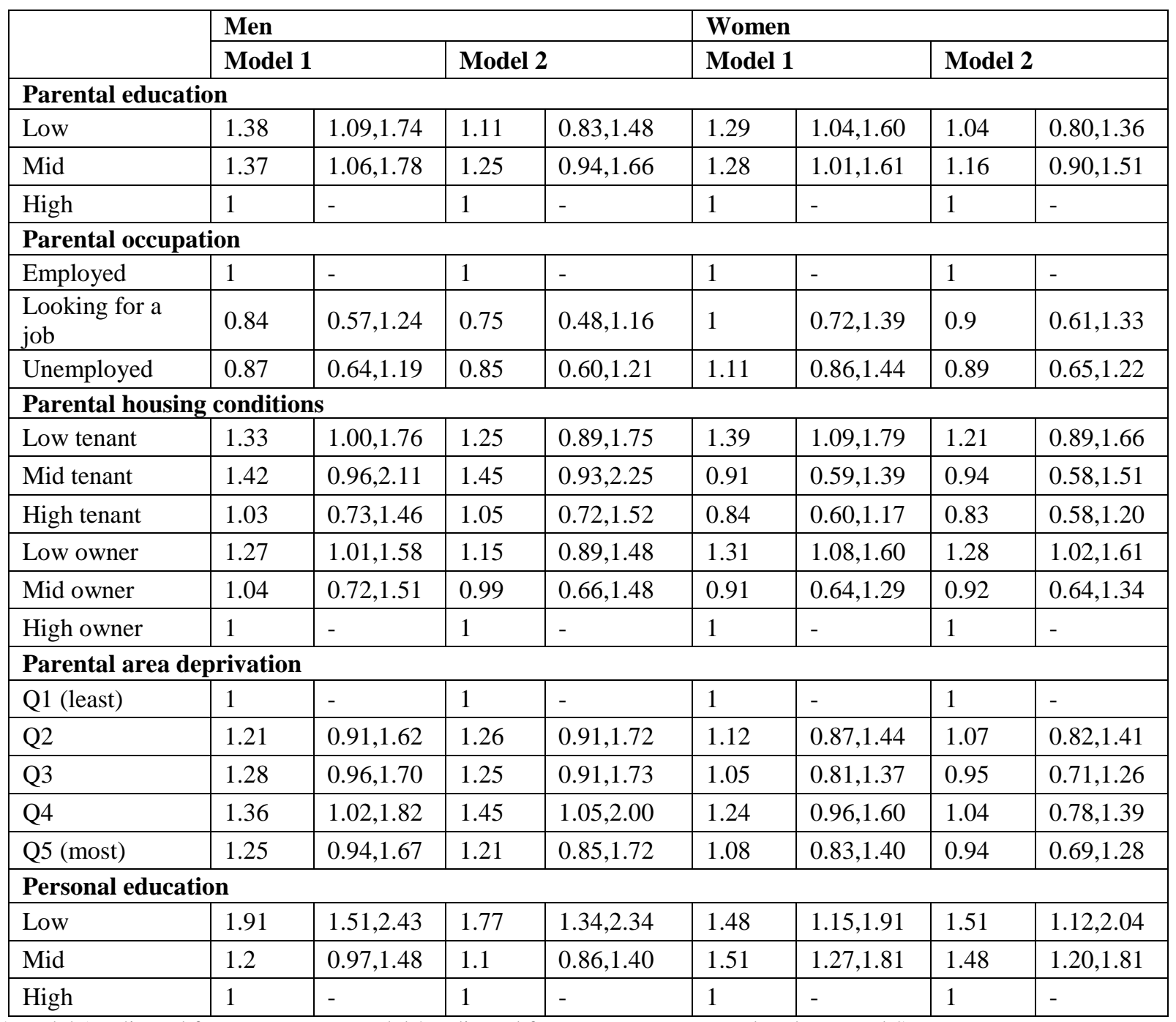

*Model 1: adjusted for current age; Model 2: adjusted for current age, parental and personal SEP.

Table 3: Relative all-cancer mortality inequalities (MRRs) by parental and personal SEP among Belgian young adults, 2001-2011. 
When looking at site-specific cancer mortality, SE inequalities are less pronounced, especially in men (Table 4, model 1). Having low-educated parents results in higher mortality from colorectal cancer and leukaemia for Belgian young adult men. The same holds true for personal education: low-educated men are more likely to die from colorectal cancer (MRR: 3.32; 95\% C.I.: 1.09-10.14), and leukaemia (MRR: 2.23; 95\% C.I.: 1.13-4.42). However, the effect on leukaemia might be due to a health selection effect because the association with personal education disappears after adjustment for SRH (results not shown). In contrast, parental education is not associated with cancer mortality among Belgian young women, but personal education is (Table 5, model 1). High-educated young women have a lower likelihood to die from cancer of the cervix, ovary, brain and from leukaemia. The associations with ovarian cancer and leukaemia however are no longer significant after adjusting for SRH (results not shown). Other important determinants of female young adult cancer mortality are the housing conditions during childhood and the deprivation of the area they lived in. For example, women who grew up in a rented house of poor quality have a MRR to die from cervical cancer of 6.25 (95\% C.I.: 2.24-17.42). Area deprivation is associated with mortality from cancers of the brain and breast. Moreover, women with unemployed parents have a likelihood to die from brain cancer that is twice as high compared to daughters from employed parents (MRR: 2.08; 95\% C.I.: 1.10-3.91).

\subsection{What is the relative importance of parental and personal SEP in explaining cancer mortality inequalities?}

In the second model, we included all parental and personal SEP indicators at once. By doing this, parental education and housing conditions are no longer associated with overall cancer mortality in men (Table 3, model 2). Living in a deprived area (Q4) is still associated with overall cancer mortality and so is personal education. Low-educated men have a 77\% (95\% C.I.: 1.34-2.34) higher mortality rate compared with high-educated men, after adjusting for all SEP indicators. Also for women, parental education is no longer associated with overall cancer mortality (Table 3, model 2). Both low- and mid-educated women have an overall cancer MR that is about $50 \%$ higher compared with high-educated women. However, after adjusting for SRH, low-educated women no longer have a higher overall cancer mortality rate, possibly pointing to a health selection effect. Moreover, women whose parents were owners of a low-quality dwelling have an overall cancer MRR of 1.28 (95\% C.I.: 1.02-1.61), adjusted for all other SEP indicators.

By adding all SEP indicators in one model, both parental education and occupation are no longer (or not) associated with site-specific cancer mortality in young adults (Tables 4 and 5, model 2). The only exception is non-Hodgkin lymphoma in men, which shows higher mortality for sons of low-educated parents (MRR; 3.82; 95\% C.I. 1.2511.57). Poor housing conditions remains associated with higher mortality from cervical cancer. Contrary to the first model, housing conditions are, after adjustment for the other SEP indicators, associated with mortality from cancers of the lung, brain and non-Hodgkin lymphoma in men, and cancers of the soft tissue, breast, and non-Hodgkin lymphoma in women. Growing up in a deprived area results in higher mortality from non-Hodgkin lymphoma and leukaemia in men, and cancers of the brain and soft tissue in women. In men, after adjustment for the other SEP indicators, colorectal cancer is no longer associated with personal education, while leukaemia still is: low-educated men have a MRR of 2.03 (95\% C.I.: 1.06-3.88) compared with high-educated men. In women, personal education is 
a stronger determinant of site-specific mortality. Being high educated is associated with lower mortality from cancers of the cervix, brain, melanoma and leukaemia. The sensitivity analyses however indicate a possible health selection effect for mortality from leukaemia (both in men and women), and for female brain cancer mortality.

\begin{tabular}{|c|c|c|c|c|c|c|c|c|c|c|c|c|}
\hline & \multicolumn{4}{|c|}{ Colorectal } & \multicolumn{4}{|c|}{ Lung } & \multicolumn{4}{|c|}{ Bone } \\
\hline & \multicolumn{2}{|c|}{ Model 1} & \multicolumn{2}{|c|}{ Model 2} & \multicolumn{2}{|c|}{ Model 1} & \multicolumn{2}{|c|}{ Model 2} & \multicolumn{2}{|c|}{ Model 1} & \multicolumn{2}{|c|}{ Model 2} \\
\hline \multicolumn{13}{|c|}{ Parental education } \\
\hline Low & 4.55 & $\begin{array}{l}1.03,20.0 \\
5\end{array}$ & $\begin{array}{l}3.1 \\
6 \\
\end{array}$ & $\begin{array}{l}0.81,12 . \\
38\end{array}$ & $\begin{array}{l}1.2 \\
7\end{array}$ & $\begin{array}{l}0.49,3.2 \\
7\end{array}$ & 0.95 & $\begin{array}{l}0.35,2.5 \\
7\end{array}$ & $\begin{array}{l}1.0 \\
5\end{array}$ & $\begin{array}{l}0.46,2.4 \\
1\end{array}$ & 1.02 & $\begin{array}{l}0.44,2 . \\
37\end{array}$ \\
\hline Mid & 2.91 & $\begin{array}{l}0.59,14.4 \\
1 \\
\end{array}$ & $\begin{array}{l}2.4 \\
6 \\
\end{array}$ & \begin{tabular}{|l|}
$0.61,9.9$ \\
8 \\
\end{tabular} & $\begin{array}{l}1.4 \\
4 \\
\end{array}$ & $\begin{array}{l}0.51,4.0 \\
5 \\
\end{array}$ & 0.75 & $\begin{array}{l}0.26,2.1 \\
7 \\
\end{array}$ & $\begin{array}{l}0.6 \\
6 \\
\end{array}$ & $\begin{array}{l}0.23,1.8 \\
5 \\
\end{array}$ & 0.64 & $\begin{array}{l}0.26,1 . \\
59\end{array}$ \\
\hline High & 1 & - & 1 & - & 1 & - & 1 & - & 1 & - & 1 & - \\
\hline \multicolumn{13}{|c|}{ Parental occupation } \\
\hline $\begin{array}{l}\text { Emplo } \\
\text { yed }\end{array}$ & 1 & - & 1 & - & 1 & - & 1 & - & 1 & - & 1 & - \\
\hline $\begin{array}{l}\text { Lookin } \\
\mathrm{g} \text { for a } \\
\text { job }\end{array}$ & 0.6 & $0.08,4.41$ & $\begin{array}{l}0.3 \\
9\end{array}$ & $\begin{array}{l}0.07,2.2 \\
5\end{array}$ & $\begin{array}{l}0.9 \\
4\end{array}$ & $\begin{array}{l}0.22,3.9 \\
5\end{array}$ & 0.52 & $\begin{array}{l}0.09,3.0 \\
6\end{array}$ & $\begin{array}{l}0.9 \\
1\end{array}$ & $\begin{array}{l}0.22,3.8 \\
3\end{array}$ & 1.83 & $\begin{array}{l}0.52,6 . \\
35\end{array}$ \\
\hline $\begin{array}{l}\text { Unemp } \\
\text { loyed }\end{array}$ & 0.7 & $0.16,3.02$ & $\begin{array}{l}0.5 \\
4 \\
\end{array}$ & \begin{tabular}{|l|}
$0.15,1.9$ \\
6 \\
\end{tabular} & $\begin{array}{l}0.5 \\
1 \\
\end{array}$ & $\begin{array}{l}0.12,2.1 \\
8 \\
\end{array}$ & 0.3 & $\begin{array}{l}0.05,1.7 \\
3 \\
\end{array}$ & $\begin{array}{l}0.7 \\
3 \\
\end{array}$ & $\begin{array}{l}0.17,3.1 \\
3 \\
\end{array}$ & 1.32 & $\begin{array}{l}0.39,4 . \\
40\end{array}$ \\
\hline \multicolumn{13}{|c|}{ Parental housing conditions } \\
\hline $\begin{array}{l}\text { Low } \\
\text { tenant }\end{array}$ & 2.49 & $0.91,6.83$ & $\begin{array}{l}1.4 \\
7 \\
\end{array}$ & \begin{tabular}{|l|}
$0.54,4.0$ \\
1
\end{tabular} & 2.1 & $\begin{array}{l}0.79,5.6 \\
3\end{array}$ & 2.99 & $\begin{array}{l}1.04,8.5 \\
9\end{array}$ & $\begin{array}{l}0.8 \\
2\end{array}$ & $\begin{array}{l}0.28,2.4 \\
0\end{array}$ & 0.48 & $\begin{array}{l}0.13,1 . \\
72\end{array}$ \\
\hline $\begin{array}{l}\text { Mid } \\
\text { tenant }\end{array}$ & 2.02 & $0.44,9.23$ & 1.5 & \begin{tabular}{|l|}
$0.39,5.8$ \\
4
\end{tabular} & - & - & - & - & $\begin{array}{l}0.5 \\
1\end{array}$ & $\begin{array}{l}0.07,3.7 \\
9\end{array}$ & 0.59 & $\begin{array}{l}0.11,3 . \\
16\end{array}$ \\
\hline $\begin{array}{l}\text { High } \\
\text { tenant }\end{array}$ & 0.54 & $0.07,4.28$ & $\begin{array}{l}0.4 \\
3 \\
\end{array}$ & \begin{tabular}{|l|}
$0.07,2.5$ \\
3 \\
\end{tabular} & $\begin{array}{l}1.3 \\
9 \\
\end{array}$ & $\begin{array}{l}0.39,4.9 \\
4\end{array}$ & 0.66 & $\begin{array}{l}0.11,3.9 \\
2\end{array}$ & $\begin{array}{l}0.5 \\
3 \\
\end{array}$ & $\begin{array}{l}0.12,2.3 \\
0\end{array}$ & 0.66 & $\begin{array}{l}0.20,2 . \\
16\end{array}$ \\
\hline $\begin{array}{l}\text { Low } \\
\text { owner }\end{array}$ & 0.79 & $0.25,2.53$ & $\begin{array}{l}0.4 \\
3 \\
\end{array}$ & \begin{tabular}{|l|}
$0.14,1.3$ \\
2
\end{tabular} & $\begin{array}{l}0.9 \\
9 \\
\end{array}$ & $\begin{array}{l}0.37,2.6 \\
4\end{array}$ & 1.21 & $\begin{array}{l}0.45,3.2 \\
3\end{array}$ & $\begin{array}{l}0.5 \\
1 \\
\end{array}$ & $\begin{array}{l}0.19,1.3 \\
7\end{array}$ & 0.5 & $\begin{array}{l}0.20,1 . \\
25\end{array}$ \\
\hline $\begin{array}{l}\text { Mid } \\
\text { owner }\end{array}$ & 1.23 & $0.27,5.59$ & $\begin{array}{l}1.0 \\
2 \\
\end{array}$ & \begin{tabular}{|l|}
$0.28,3.7$ \\
5 \\
\end{tabular} & $\begin{array}{l}1.5 \\
2 \\
\end{array}$ & $\begin{array}{l}0.43,5.3 \\
9 \\
\end{array}$ & 2.18 & $\begin{array}{l}0.70,6.7 \\
9 \\
\end{array}$ & - & - & - & - \\
\hline $\begin{array}{l}\text { High } \\
\text { owner }\end{array}$ & 1 & - & 1 & - & 1 & - & 1 & - & 1 & - & 1 & - \\
\hline \multicolumn{13}{|c|}{ Parental area deprivation } \\
\hline Q1 & 1 & - & 1 & - & 1 & - & 1 & - & 1 & - & 1 & - \\
\hline Q2 & 2.68 & $\begin{array}{l}0.52,13.8 \\
2\end{array}$ & $\begin{array}{l}2.2 \\
8 \\
\end{array}$ & \begin{tabular}{|l|}
$0.56,9.3$ \\
0 \\
\end{tabular} & $\begin{array}{l}0.4 \\
6 \\
\end{array}$ & $\begin{array}{l}0.12,1.7 \\
7\end{array}$ & 0.79 & $\begin{array}{l}0.22,2.8 \\
9\end{array}$ & 1.8 & $\begin{array}{l}0.65,4.9 \\
6\end{array}$ & 2.01 & $\begin{array}{l}0.84,4 . \\
82\end{array}$ \\
\hline Q3 & 3.34 & $\begin{array}{l}0.68,16.5 \\
1 \\
\end{array}$ & $\begin{array}{l}2.2 \\
7 \\
\end{array}$ & \begin{tabular}{|l}
$0.55,9.3$ \\
5 \\
\end{tabular} & $\begin{array}{l}1.5 \\
8 \\
\end{array}$ & $\begin{array}{l}0.60,4.1 \\
5 \\
\end{array}$ & 2.16 & $\begin{array}{l}0.75,6.2 \\
1\end{array}$ & $\begin{array}{l}0.5 \\
7 \\
\end{array}$ & $\begin{array}{l}0.14,2.2 \\
7 \\
\end{array}$ & 0.24 & $\begin{array}{l}0.04,1 . \\
34\end{array}$ \\
\hline Q4 & 4.13 & $\begin{array}{l}0.86,19.8 \\
5\end{array}$ & $\begin{array}{l}2.8 \\
4 \\
\end{array}$ & \begin{tabular}{|l|}
$0.70,11$ \\
50
\end{tabular} & $\begin{array}{l}0.8 \\
4 \\
\end{array}$ & $\begin{array}{l}0.27,2.6 \\
4 \\
\end{array}$ & 1.15 & $\begin{array}{l}0.34,3.9 \\
4\end{array}$ & 1.2 & $\begin{array}{l}0.39,3.7 \\
3 \\
\end{array}$ & 1.39 & $\begin{array}{l}0.51,3 . \\
83\end{array}$ \\
\hline Q5 & 2.79 & $\begin{array}{l}0.54,14.3 \\
6\end{array}$ & $\begin{array}{l}2.2 \\
8 \\
\end{array}$ & \begin{tabular}{|l|}
$0.52,9.9$ \\
6 \\
\end{tabular} & $\begin{array}{l}1.2 \\
7 \\
\end{array}$ & $\begin{array}{l}0.46,3.5 \\
0 \\
\end{array}$ & 1.22 & $\begin{array}{l}0.34,4.3 \\
9\end{array}$ & $\begin{array}{l}1.3 \\
2 \\
\end{array}$ & $\begin{array}{l}0.44,3.9 \\
3 \\
\end{array}$ & 1.22 & $\begin{array}{l}0.39,3 . \\
81\end{array}$ \\
\hline \multicolumn{13}{|c|}{ Personal education } \\
\hline Low & 3.32 & $\begin{array}{l}1.09,10.1 \\
4\end{array}$ & \begin{tabular}{|l|}
2.3 \\
8 \\
\end{tabular} & \begin{tabular}{|l|}
$0.85,6.7$ \\
2 \\
\end{tabular} & $\begin{array}{l}2.0 \\
6 \\
\end{array}$ & $\begin{array}{l}0.85,4.9 \\
4 \\
\end{array}$ & 1.33 & $\begin{array}{l}0.49,3.6 \\
1\end{array}$ & $\begin{array}{l}0.6 \\
3\end{array}$ & $\begin{array}{l}0.17,2.2 \\
9\end{array}$ & 0.62 & $\begin{array}{l}0.17,2 . \\
27\end{array}$ \\
\hline Mid & 1.71 & $0.58,4.99$ & 1.3 & \begin{tabular}{|l|}
$0.50,3.3$ \\
8 \\
\end{tabular} & \begin{tabular}{|l|}
0.7 \\
7 \\
\end{tabular} & $\begin{array}{l}0.31,1.9 \\
0 \\
\end{array}$ & 0.73 & $\begin{array}{l}0.30,1.7 \\
6 \\
\end{array}$ & $\begin{array}{l}1.5 \\
9 \\
\end{array}$ & $\begin{array}{l}0.74,3.4 \\
2 \\
\end{array}$ & 1.65 & $\begin{array}{l}0.79,3 . \\
47 \\
\end{array}$ \\
\hline High & 1 & - & 1 & - & 1 & - & 1 & - & 1 & - & 1 & - \\
\hline
\end{tabular}




\begin{tabular}{|c|c|c|c|c|c|c|c|c|c|c|c|c|}
\hline & \multicolumn{4}{|c|}{ Melanoma } & \multicolumn{5}{|c|}{ Brain } & \multicolumn{3}{|c|}{ Non-Hodgkin } \\
\hline & \multicolumn{2}{|c|}{ Model 1} & \multicolumn{2}{|c|}{ Model 2} & \multicolumn{2}{|c|}{ Model 1} & \multicolumn{2}{|c|}{ Model 2} & \multicolumn{2}{|c|}{ Model 1} & \multicolumn{2}{|c|}{ Model 2} \\
\hline \multicolumn{13}{|c|}{ Parental education } \\
\hline Low & 1.2 & $0.59,2.44$ & $\begin{array}{l}1.4 \\
6 \\
\end{array}$ & $\begin{array}{l}0.72,2.9 \\
9\end{array}$ & 0.8 & $\begin{array}{l}0.47,1.3 \\
6 \\
\end{array}$ & 0.7 & $\begin{array}{l}0.42,1.1 \\
8 \\
\end{array}$ & $\begin{array}{l}2.3 \\
6 \\
\end{array}$ & $\begin{array}{l}0.89,6.2 \\
6 \\
\end{array}$ & 3.8 & $\begin{array}{l}1.25,11 \\
.57\end{array}$ \\
\hline Mid & 1.33 & $0.61,2.89$ & $\begin{array}{l}1.5 \\
9 \\
\end{array}$ & $\begin{array}{l}0.80,3.1 \\
7 \\
\end{array}$ & 1.1 & $\begin{array}{l}0.63,1.9 \\
3 \\
\end{array}$ & 1 & $\begin{array}{l}0.62,1.6 \\
3 \\
\end{array}$ & $\begin{array}{l}1.1 \\
8 \\
\end{array}$ & $\begin{array}{l}0.36,3.8 \\
6 \\
\end{array}$ & 2.01 & $\begin{array}{l}0.61,6 . \\
56\end{array}$ \\
\hline High & 1 & - & 1 & - & 1 & - & 1 & - & 1 & - & 1 & - \\
\hline \multicolumn{13}{|c|}{ Parental occupation } \\
\hline $\begin{array}{l}\text { Emplo } \\
\text { yed }\end{array}$ & 1 & - & 1 & - & 1 & - & 1 & - & 1 & - & 1 & - \\
\hline $\begin{array}{l}\text { Lookin } \\
\mathrm{g} \text { for a } \\
\text { job }\end{array}$ & 0.89 & $0.28,2.86$ & $\begin{array}{l}1.1 \\
6\end{array}$ & $\begin{array}{l}0.41,3.2 \\
6\end{array}$ & $\begin{array}{l}0.5 \\
3\end{array}$ & $\begin{array}{l}0.17,1.6 \\
7\end{array}$ & 0.43 & $\begin{array}{l}0.14,1.3 \\
6\end{array}$ & $\begin{array}{l}0.8 \\
9\end{array}$ & $\begin{array}{l}0.21,3.7 \\
3\end{array}$ & 0.82 & $\begin{array}{l}0.23,2 . \\
94\end{array}$ \\
\hline $\begin{array}{l}\text { Unemp } \\
\text { loyed }\end{array}$ & 0.74 & $0.26,2.06$ & $\begin{array}{l}0.8 \\
9 \\
\end{array}$ & $\begin{array}{l}0.36,2.1 \\
6\end{array}$ & \begin{tabular}{|l|}
0.6 \\
8 \\
\end{tabular} & $\begin{array}{l}0.30,1.5 \\
4\end{array}$ & 0.56 & $\begin{array}{l}0.24,1.2 \\
7\end{array}$ & $\begin{array}{l}0.3 \\
1 \\
\end{array}$ & $\begin{array}{l}0.04,2.3 \\
2\end{array}$ & 0.29 & $\begin{array}{l}0.05,1 . \\
62\end{array}$ \\
\hline \multicolumn{13}{|c|}{ Parental housing conditions } \\
\hline $\begin{array}{l}\text { Low } \\
\text { tenant }\end{array}$ & 0.76 & $0.29,1.96$ & $\begin{array}{l}0.7 \\
5\end{array}$ & $\begin{array}{l}0.29,1.9 \\
4\end{array}$ & 1.4 & $\begin{array}{l}0.71,2.7 \\
9\end{array}$ & 1.79 & $\begin{array}{l}0.94,3.4 \\
2\end{array}$ & $\begin{array}{l}0.5 \\
7\end{array}$ & $\begin{array}{l}0.13,2.5 \\
1\end{array}$ & 0.36 & $\begin{array}{l}0.10,1 . \\
33\end{array}$ \\
\hline $\begin{array}{l}\text { Mid } \\
\text { tenant }\end{array}$ & 0.74 & $0.18,3.12$ & $\begin{array}{l}0.8 \\
9 \\
\end{array}$ & $\begin{array}{l}0.26,3.1 \\
1 \\
\end{array}$ & - & - & - & - & $\begin{array}{l}2.1 \\
1 \\
\end{array}$ & $\begin{array}{l}0.61,7.3 \\
4 \\
\end{array}$ & 1.38 & $\begin{array}{l}0.45,4 . \\
25\end{array}$ \\
\hline $\begin{array}{l}\text { High } \\
\text { tenant }\end{array}$ & 0.6 & $0.18,1.96$ & 0.7 & $\begin{array}{l}0.26,1.9 \\
2\end{array}$ & \begin{tabular}{|l|}
1.8 \\
4 \\
\end{tabular} & $\begin{array}{l}0.92,3.6 \\
5 \\
\end{array}$ & 2.2 & $\begin{array}{l}1.25,3.8 \\
7\end{array}$ & $\begin{array}{l}0.7 \\
4 \\
\end{array}$ & $\begin{array}{l}0.17,3.2 \\
5\end{array}$ & 0.57 & $\begin{array}{l}0.16,2 . \\
02\end{array}$ \\
\hline $\begin{array}{l}\text { Low } \\
\text { owner }\end{array}$ & 0.8 & $0.40,1.62$ & $\begin{array}{l}0.8 \\
6 \\
\end{array}$ & $\begin{array}{l}0.45,1.6 \\
4\end{array}$ & 1.6 & $\begin{array}{l}0.95,2.6 \\
9\end{array}$ & 1.71 & $\begin{array}{l}1.07,2.7 \\
2\end{array}$ & $\begin{array}{l}1.1 \\
2 \\
\end{array}$ & $\begin{array}{l}0.47,2.6 \\
6 \\
\end{array}$ & 0.38 & $\begin{array}{l}0.15,0 . \\
99\end{array}$ \\
\hline $\begin{array}{l}\text { Mid } \\
\text { owner }\end{array}$ & 0.91 & $0.32,2.61$ & $\begin{array}{l}1.0 \\
2 \\
\end{array}$ & $\begin{array}{l}0.42,2.4 \\
8 \\
\end{array}$ & \begin{tabular}{|l|}
0.9 \\
7 \\
\end{tabular} & $\begin{array}{l}0.38,2.4 \\
8 \\
\end{array}$ & 1.16 & $\begin{array}{l}0.54,2.5 \\
0\end{array}$ & $\begin{array}{l}1.3 \\
4 \\
\end{array}$ & $\begin{array}{l}0.38,4.6 \\
5 \\
\end{array}$ & 0.36 & $\begin{array}{l}0.06,1 . \\
97\end{array}$ \\
\hline $\begin{array}{l}\text { High } \\
\text { owner }\end{array}$ & 1 & - & 1 & - & 1 & - & 1 & - & 1 & - & 1 & - \\
\hline \multicolumn{13}{|c|}{ Parental area deprivation } \\
\hline Q1 & 1 & - & 1 & - & 1 & - & 1 & - & 1 & - & 1 & - \\
\hline Q2 & 1.15 & $0.56,2.39$ & $\begin{array}{l}1.0 \\
4 \\
\end{array}$ & $\begin{array}{l}0.54,1.9 \\
8\end{array}$ & \begin{tabular}{|l|}
0.9 \\
6 \\
\end{tabular} & $\begin{array}{l}0.49,1.8 \\
7 \\
\end{array}$ & 1 & $\begin{array}{l}0.57,1.7 \\
3\end{array}$ & $\begin{array}{l}1.3 \\
5 \\
\end{array}$ & $\begin{array}{l}0.36,5.0 \\
2\end{array}$ & 1.96 & $\begin{array}{l}0.47,8 \\
18\end{array}$ \\
\hline Q3 & 0.64 & $0.27,1.52$ & $\begin{array}{l}0.5 \\
8 \\
\end{array}$ & $\begin{array}{l}0.27,1.2 \\
6\end{array}$ & 1 & $\begin{array}{l}0.51,1.9 \\
5\end{array}$ & 0.97 & $\begin{array}{l}0.55,1.7 \\
1\end{array}$ & $\begin{array}{l}1.9 \\
7 \\
\end{array}$ & $\begin{array}{l}0.58,6.7 \\
3 \\
\end{array}$ & 3.08 & $\begin{array}{l}0.80,11 \\
.91\end{array}$ \\
\hline Q4 & 0.51 & $0.19,1.32$ & $\begin{array}{l}0.5 \\
5\end{array}$ & $\begin{array}{l}0.24,1.2 \\
5\end{array}$ & \begin{tabular}{|l|}
1.2 \\
5
\end{tabular} & $\begin{array}{l}0.66,2.3 \\
9\end{array}$ & 1.26 & $\begin{array}{l}0.72,2.1 \\
8\end{array}$ & $\begin{array}{l}2.0 \\
9\end{array}$ & $\begin{array}{l}0.61,7.1 \\
3\end{array}$ & 4.15 & $\begin{array}{l}1.09,15 \\
.83\end{array}$ \\
\hline Q5 & 0.72 & $0.31,1.66$ & $\begin{array}{l}0.7 \\
7 \\
\end{array}$ & $\begin{array}{l}0.34,1.7 \\
1\end{array}$ & \begin{tabular}{|l|}
0.7 \\
5 \\
\end{tabular} & $\begin{array}{l}0.36,1.5 \\
5\end{array}$ & 0.82 & $\begin{array}{l}0.42,1.5 \\
9\end{array}$ & $\begin{array}{l}1.9 \\
7 \\
\end{array}$ & $\begin{array}{l}0.58,6.7 \\
2\end{array}$ & 4.71 & $\begin{array}{l}1.19,18 \\
.65\end{array}$ \\
\hline \multicolumn{13}{|c|}{ Personal education } \\
\hline Low & 1.39 & $0.67,2.88$ & $\begin{array}{l}1.3 \\
9 \\
\end{array}$ & $\begin{array}{l}0.70,2.7 \\
6 \\
\end{array}$ & \begin{tabular}{|l|}
1.1 \\
9 \\
\end{tabular} & $\begin{array}{l}0.65,2.2 \\
0 \\
\end{array}$ & 1.48 & $\begin{array}{l}0.83,2.6 \\
2\end{array}$ & $\begin{array}{l}0.9 \\
3 \\
\end{array}$ & $\begin{array}{l}0.29,3.0 \\
3 \\
\end{array}$ & 0.71 & $\begin{array}{l}0.24,2 . \\
05\end{array}$ \\
\hline Mid & 0.99 & $0.53,1.87$ & $\begin{array}{l}0.8 \\
5 \\
\end{array}$ & $\begin{array}{l}0.48,1.5 \\
2 \\
\end{array}$ & \begin{tabular}{|l|}
1.1 \\
9 \\
\end{tabular} & $\begin{array}{l}0.73,1.9 \\
3 \\
\end{array}$ & 1.46 & $\begin{array}{l}0.95,2.2 \\
7 \\
\end{array}$ & 1.5 & $\begin{array}{l}0.66,3.3 \\
9 \\
\end{array}$ & 1.1 & $\begin{array}{l}0.51,2 . \\
38\end{array}$ \\
\hline \multirow[t]{3}{*}{ High } & 1 & - & 1 & - & 1 & - & 1 & - & 1 & - & 1 & - \\
\hline & \multicolumn{8}{|c|}{ Leukaemia } & & & & \\
\hline & \multicolumn{4}{|c|}{ Model 1} & \multicolumn{4}{|c|}{ Model 2} & & & & \\
\hline \multicolumn{9}{|c|}{ Parental education } & & & & \\
\hline Low & 2 & & $2,3.9$ & & 1.08 & & $55,2.12$ & & & & & \\
\hline Mid & 1.7 & & $36,3.7$ & & 1.53 & & $81,2.92$ & & & & & \\
\hline High & 1 & - & & & 1 & - & & & & & & \\
\hline
\end{tabular}




\begin{tabular}{|c|c|c|c|c|}
\hline \multicolumn{5}{|c|}{ Parental occupation } \\
\hline Employed & 1 & - & 1 & - \\
\hline $\begin{array}{l}\text { Looking for } \\
\text { a job }\end{array}$ & 1.46 & $0.63,3.39$ & 1.12 & $0.46,2.69$ \\
\hline Unemployed & 1.79 & $0.91,3.51$ & 1.7 & $0.89,3.27$ \\
\hline \multicolumn{5}{|c|}{ Parental housing conditions } \\
\hline Low tenant & 1.37 & $0.64,2.93$ & 0.77 & $0.35,1.70$ \\
\hline Mid tenant & 1.88 & $0.72,4.90$ & 0.96 & $0.35,2.68$ \\
\hline High tenant & 0.8 & $0.28,2.28$ & 0.56 & $0.21,1.52$ \\
\hline Low owner & 1.58 & $0.89,2.80$ & 1.23 & $0.72,2.08$ \\
\hline Mid owner & 1.44 & $0.59,3.51$ & 1.19 & $0.53,2.64$ \\
\hline High owner & 1 & - & 1 & - \\
\hline \multicolumn{5}{|c|}{ Parental area deprivation } \\
\hline Q1 & 1 & - & 1 & - \\
\hline Q2 & 1.08 & $0.45,2.59$ & 1.28 & $0.57,2.87$ \\
\hline Q3 & 1.81 & $0.82,3.98$ & 2.18 & $1.04,4.58$ \\
\hline Q4 & 2.15 & $0.99,4.67$ & 2.51 & $1.19,5.29$ \\
\hline Q5 & 1.8 & $0.82,3.98$ & 1.73 & $0.76,3.94$ \\
\hline \multicolumn{5}{|c|}{ Personal education } \\
\hline Low & 2.23 & $1.13,4.42$ & 2.03 & $1.06,3.88$ \\
\hline Mid & 1.68 & $0.92,3.07$ & 1.57 & $0.91,2.72$ \\
\hline High & 1 & - & 1 & - \\
\hline
\end{tabular}

* Model 1: adjusted for current age; Model 2: adjusted for current age, parental and personal SEP.

Table 4: Relative site-specific cancer mortality inequalities (MRRs) by parental and personal SEP among Belgian male young adults, 2001-2011.

\begin{tabular}{|c|c|c|c|c|c|c|c|c|c|c|c|c|}
\hline & \multicolumn{4}{|c|}{ Colorectal } & \multicolumn{4}{|c|}{ Melanoma } & \multicolumn{4}{|c|}{ Soft tissue } \\
\hline & \multicolumn{2}{|c|}{ Model 1} & \multicolumn{2}{|c|}{ Model 2} & \multicolumn{2}{|c|}{ Model 1} & \multicolumn{2}{|c|}{ Model 2} & \multicolumn{2}{|c|}{ Model 1} & \multicolumn{2}{|c|}{ Model 2} \\
\hline \multicolumn{13}{|c|}{ Parental education } \\
\hline Low & $\begin{array}{l}1.0 \\
5\end{array}$ & $\begin{array}{l}0.36,3 . \\
06\end{array}$ & $\begin{array}{l}0.8 \\
9\end{array}$ & $\begin{array}{l}0.27,2 . \\
92\end{array}$ & $\begin{array}{l}0.7 \\
5\end{array}$ & $\begin{array}{l}0.35,1.6 \\
1\end{array}$ & $\begin{array}{l}0.5 \\
9\end{array}$ & $\begin{array}{l}0.27,1.3 \\
0\end{array}$ & $\begin{array}{l}1.0 \\
6\end{array}$ & $\begin{array}{l}0.47,2 . \\
42\end{array}$ & $\begin{array}{l}1.1 \\
2\end{array}$ & $\begin{array}{l}0.48,2.5 \\
9\end{array}$ \\
\hline Mid & $\begin{array}{l}2.6 \\
9\end{array}$ & $\begin{array}{l}0.97,7 . \\
47\end{array}$ & $\begin{array}{l}2.2 \\
1\end{array}$ & $\begin{array}{l}0.78,6 . \\
24\end{array}$ & $\begin{array}{l}0.7 \\
3\end{array}$ & $\begin{array}{l}0.31,1.7 \\
3\end{array}$ & $\begin{array}{l}0.5 \\
9\end{array}$ & $\begin{array}{l}0.26,1.3 \\
0\end{array}$ & $\begin{array}{l}0.8 \\
7\end{array}$ & $\begin{array}{l}0.33,2 . \\
25\end{array}$ & $\begin{array}{l}0.8 \\
2\end{array}$ & $\begin{array}{l}0.34,1.9 \\
7\end{array}$ \\
\hline High & 1 & - & 1 & - & 1 & - & 1 & - & 1 & - & 1 & - \\
\hline \multicolumn{13}{|c|}{ Parental occupation } \\
\hline Employed & 1 & - & 1 & - & 1 & - & 1 & - & 1 & - & 1 & - \\
\hline $\begin{array}{l}\text { Looking } \\
\text { for a job }\end{array}$ & 1 & $\begin{array}{l}0.24,4 . \\
20\end{array}$ & $\begin{array}{l}1.8 \\
2\end{array}$ & $\begin{array}{l}0.39,8 . \\
38\end{array}$ & $\begin{array}{l}1.2 \\
4\end{array}$ & $\begin{array}{l}0.38,4.0 \\
5\end{array}$ & $\begin{array}{l}1.2 \\
2\end{array}$ & $\begin{array}{l}0.34,4.4 \\
3\end{array}$ & $\begin{array}{l}0.4 \\
2\end{array}$ & $\begin{array}{l}0.06,3 . \\
08\end{array}$ & $\begin{array}{l}0.6 \\
5\end{array}$ & $\begin{array}{l}0.12,3.6 \\
5\end{array}$ \\
\hline $\begin{array}{l}\text { Unemploy } \\
\text { ed }\end{array}$ & $\begin{array}{l}0.5 \\
8\end{array}$ & $\begin{array}{l}0.14,2 . \\
46\end{array}$ & $\begin{array}{l}0.9 \\
3\end{array}$ & $\begin{array}{l}0.21,4 . \\
08\end{array}$ & $\begin{array}{l}1.0 \\
9\end{array}$ & $\begin{array}{l}0.38,3.1 \\
3\end{array}$ & $\begin{array}{l}0.7 \\
2\end{array}$ & $\begin{array}{l}0.20,2.5 \\
4\end{array}$ & $\begin{array}{l}0.9 \\
4\end{array}$ & $\begin{array}{l}0.29,3 . \\
07\end{array}$ & $\begin{array}{l}0.4 \\
3\end{array}$ & $\begin{array}{l}0.08,2.3 \\
3\end{array}$ \\
\hline
\end{tabular}




\begin{tabular}{|c|c|c|c|c|c|c|c|c|c|c|c|c|}
\hline \multicolumn{13}{|c|}{ Parental housing conditions } \\
\hline $\begin{array}{l}\text { Low } \\
\text { tenant }\end{array}$ & $\begin{array}{l}0.2 \\
6\end{array}$ & $\begin{array}{l}0.03,1 . \\
95\end{array}$ & - & - & \begin{tabular}{|l|}
0.9 \\
5
\end{tabular} & $\begin{array}{l}0.32,2.8 \\
1\end{array}$ & $\begin{array}{l}0.8 \\
4\end{array}$ & $\begin{array}{l}0.27,2.6 \\
6\end{array}$ & $\begin{array}{l}1.6 \\
8\end{array}$ & $\begin{array}{l}0.65,4 . \\
37\end{array}$ & $\begin{array}{l}2.6 \\
4\end{array}$ & $\begin{array}{l}1.03,6.8 \\
2\end{array}$ \\
\hline Mid tenant & $\begin{array}{l}1.2 \\
2\end{array}$ & $\begin{array}{l}0.28,5 \\
30\end{array}$ & $\begin{array}{l}1.2 \\
8\end{array}$ & $\begin{array}{l}0.27,6 . \\
16\end{array}$ & - & - & - & - & - & - & - & - \\
\hline $\begin{array}{l}\text { High } \\
\text { tenant }\end{array}$ & - & - & - & - & \begin{tabular}{|l|}
0.9 \\
2
\end{tabular} & $\begin{array}{l}0.27,3.1 \\
4\end{array}$ & $\begin{array}{l}0.9 \\
7\end{array}$ & $\begin{array}{l}0.33,2.8 \\
2\end{array}$ & $\begin{array}{l}1.0 \\
8\end{array}$ & $\begin{array}{l}0.31,3 . \\
77\end{array}$ & $\begin{array}{l}0.4 \\
9\end{array}$ & $\begin{array}{l}0.09,2.7 \\
1\end{array}$ \\
\hline $\begin{array}{l}\text { Low } \\
\text { owner }\end{array}$ & 1 & $\begin{array}{l}0.43,2 . \\
33\end{array}$ & $\begin{array}{l}0.9 \\
2\end{array}$ & $\begin{array}{l}0.36,2 . \\
35\end{array}$ & \begin{tabular}{|l|}
1.2 \\
8
\end{tabular} & $\begin{array}{l}0.60,2.7 \\
3\end{array}$ & $\begin{array}{l}1.1 \\
8\end{array}$ & $\begin{array}{l}0.57,2.4 \\
7\end{array}$ & $\begin{array}{l}1.2 \\
5\end{array}$ & $\begin{array}{l}0.54,2 . \\
89\end{array}$ & 1.3 & $\begin{array}{l}0.58,2.8 \\
8\end{array}$ \\
\hline $\begin{array}{l}\text { Mid } \\
\text { owner }\end{array}$ & $\begin{array}{l}1.1 \\
5\end{array}$ & $\begin{array}{l}0.34,3 . \\
96\end{array}$ & $\begin{array}{l}1.1 \\
7 \\
\end{array}$ & $\begin{array}{l}0.34,4 . \\
03\end{array}$ & \begin{tabular}{|l|}
0.7 \\
3 \\
\end{tabular} & $\begin{array}{l}0.17,3.1 \\
6\end{array}$ & $\begin{array}{l}0.7 \\
9 \\
\end{array}$ & $\begin{array}{l}0.22,2.7 \\
9\end{array}$ & $\begin{array}{l}0.8 \\
9\end{array}$ & $\begin{array}{l}0.20,3 . \\
93\end{array}$ & $\begin{array}{l}1.0 \\
7\end{array}$ & $\begin{array}{l}0.31,3.7 \\
3\end{array}$ \\
\hline $\begin{array}{l}\text { High } \\
\text { owner }\end{array}$ & 1 & - & 1 & - & 1 & - & 1 & - & 1 & - & 1 & - \\
\hline \multicolumn{13}{|c|}{ Parental area deprivation } \\
\hline Q1 & 1 & - & 1 & - & 1 & - & 1 & - & 1 & - & 1 & - \\
\hline Q2 & $\begin{array}{l}1.2 \\
2\end{array}$ & $\begin{array}{l}0.44,3 . \\
37\end{array}$ & $\begin{array}{l}1.1 \\
8\end{array}$ & $\begin{array}{l}0.43,3 . \\
20\end{array}$ & \begin{tabular}{|l|}
0.9 \\
5
\end{tabular} & $\begin{array}{l}0.37,2.4 \\
7\end{array}$ & $\begin{array}{l}0.9 \\
8\end{array}$ & $\begin{array}{l}0.43,2.2 \\
3\end{array}$ & $\begin{array}{l}2.7 \\
9\end{array}$ & $\begin{array}{l}1.00,7 . \\
84\end{array}$ & $\begin{array}{l}2.6 \\
2\end{array}$ & $\begin{array}{l}1.09,6.2 \\
6\end{array}$ \\
\hline Q3 & $\begin{array}{l}0.3 \\
2\end{array}$ & $\begin{array}{l}0.07,1 . \\
54\end{array}$ & $\begin{array}{l}0.3 \\
2\end{array}$ & $\begin{array}{l}0.07,1 . \\
51\end{array}$ & 1 & $\begin{array}{l}0.39,2.5 \\
9\end{array}$ & $\begin{array}{l}1.0 \\
3\end{array}$ & $\begin{array}{l}0.45,2.3 \\
8\end{array}$ & $\begin{array}{l}1.1 \\
3\end{array}$ & $\begin{array}{l}0.33,3 . \\
89\end{array}$ & $\begin{array}{l}1.0 \\
5\end{array}$ & $\begin{array}{l}0.37,3.0 \\
1\end{array}$ \\
\hline Q4 & $\begin{array}{l}1.1 \\
8\end{array}$ & $\begin{array}{l}0.41,3 . \\
35\end{array}$ & $\begin{array}{l}1.1 \\
2\end{array}$ & $\begin{array}{l}0.37,3 . \\
37\end{array}$ & \begin{tabular}{|l|}
0.9 \\
2
\end{tabular} & $\begin{array}{l}0.34,2.4 \\
7\end{array}$ & $\begin{array}{l}0.8 \\
2\end{array}$ & $\begin{array}{l}0.33,2.0 \\
6\end{array}$ & $\begin{array}{l}0.7 \\
1\end{array}$ & $\begin{array}{l}0.17,2 . \\
99\end{array}$ & $\begin{array}{l}0.6 \\
8\end{array}$ & $\begin{array}{l}0.20,2.3 \\
2\end{array}$ \\
\hline Q5 & $\begin{array}{l}0.7 \\
9 \\
\end{array}$ & $\begin{array}{l}0.25,2 . \\
50\end{array}$ & $\begin{array}{l}0.9 \\
4 \\
\end{array}$ & $\begin{array}{l}0.26,3 . \\
42\end{array}$ & \begin{tabular}{|l|}
0.3 \\
7 \\
\end{tabular} & $\begin{array}{l}0.10,1.3 \\
7\end{array}$ & $\begin{array}{l}0.4 \\
2\end{array}$ & $\begin{array}{l}0.13,1.4 \\
0\end{array}$ & $\begin{array}{l}0.6 \\
7\end{array}$ & $\begin{array}{l}0.16,2 . \\
79\end{array}$ & $\begin{array}{l}0.4 \\
8\end{array}$ & $\begin{array}{l}0.11,2.0 \\
0\end{array}$ \\
\hline \multicolumn{13}{|c|}{ Personal education } \\
\hline Low & $\begin{array}{l}0.6 \\
5\end{array}$ & $\begin{array}{l}0.15,2 . \\
88\end{array}$ & $\begin{array}{l}0.5 \\
6\end{array}$ & $\begin{array}{l}0.07,4 . \\
54\end{array}$ & \begin{tabular}{|l|}
2.0 \\
3 \\
\end{tabular} & $\begin{array}{l}0.82,4.9 \\
8\end{array}$ & $\begin{array}{l}3.4 \\
8 \\
\end{array}$ & $\begin{array}{l}1.46,8.3 \\
0\end{array}$ & 0.3 & $\begin{array}{l}0.04,2 . \\
24\end{array}$ & $\begin{array}{l}0.3 \\
7 \\
\end{array}$ & $\begin{array}{l}0.07,2.1 \\
4\end{array}$ \\
\hline Mid & $\begin{array}{l}1.6 \\
2 \\
\end{array}$ & $\begin{array}{l}0.78,3 . \\
36\end{array}$ & $\begin{array}{l}2.0 \\
7 \\
\end{array}$ & $\begin{array}{l}0.92,4 . \\
66\end{array}$ & 1.4 & $\begin{array}{l}0.69,2.8 \\
3\end{array}$ & $\begin{array}{l}1.4 \\
1 \\
\end{array}$ & $\begin{array}{l}0.70,2.8 \\
6\end{array}$ & $\begin{array}{l}1.4 \\
7\end{array}$ & $\begin{array}{l}0.74,2 . \\
94\end{array}$ & $\begin{array}{l}1.2 \\
4 \\
\end{array}$ & $\begin{array}{l}0.62,2.4 \\
6\end{array}$ \\
\hline \multirow[t]{3}{*}{ High } & 1 & - & 1 & - & 1 & - & 1 & - & 1 & - & 1 & - \\
\hline & \multicolumn{4}{|c|}{ Breast } & \multicolumn{4}{|c|}{ Cervix } & \multicolumn{4}{|c|}{ Ovary } \\
\hline & \multicolumn{2}{|c|}{ Model 1} & \multicolumn{2}{|c|}{ Model 2} & \multicolumn{2}{|c|}{ Model 1} & \multicolumn{2}{|c|}{ Model 2} & \multicolumn{2}{|c|}{ Model 1} & \multicolumn{2}{|c|}{ Model 2} \\
\hline \multicolumn{13}{|c|}{ Parental education } \\
\hline Low & $\begin{array}{l}1.4 \\
3\end{array}$ & $\begin{array}{l}0.90,2 . \\
29\end{array}$ & $\begin{array}{l}1.2 \\
3\end{array}$ & $\begin{array}{l}0.78,1 . \\
94\end{array}$ & \begin{tabular}{|l|}
3.3 \\
7 \\
\end{tabular} & $\begin{array}{l}0.75,15 \text {. } \\
11\end{array}$ & $\begin{array}{l}2.2 \\
9\end{array}$ & $\begin{array}{l}0.35,15 . \\
18\end{array}$ & $\begin{array}{l}2.0 \\
2\end{array}$ & $\begin{array}{l}0.66,6 . \\
13\end{array}$ & 2.4 & $\begin{array}{l}0.59,9.8 \\
6\end{array}$ \\
\hline Mid & $\begin{array}{l}1.4 \\
3\end{array}$ & $\begin{array}{l}0.85,2 . \\
40\end{array}$ & $\begin{array}{l}1.2 \\
9\end{array}$ & $\begin{array}{l}0.82,2 . \\
04\end{array}$ & \begin{tabular}{|l|}
4.3 \\
1
\end{tabular} & $\begin{array}{l}0.93,19 . \\
95\end{array}$ & $\begin{array}{l}5.4 \\
2\end{array}$ & $\begin{array}{l}0.87,33 . \\
83\end{array}$ & $\begin{array}{l}1.4 \\
6\end{array}$ & $\begin{array}{l}0.41,5 . \\
17\end{array}$ & $\begin{array}{l}1.9 \\
2\end{array}$ & $\begin{array}{l}0.46,7.9 \\
7\end{array}$ \\
\hline High & 1 & - & 1 & - & 1 & - & 1 & - & 1 & - & 1 & - \\
\hline \multicolumn{13}{|c|}{ Parental occupation } \\
\hline Employed & 1 & - & 1 & - & 1 & - & 1 & - & 1 & - & 1 & - \\
\hline $\begin{array}{l}\text { Looking } \\
\text { for a job }\end{array}$ & $\begin{array}{l}0.8 \\
3\end{array}$ & $\begin{array}{l}0.38,1 . \\
77\end{array}$ & 0.6 & $\begin{array}{l}0.28,1 . \\
27\end{array}$ & $\begin{array}{l}1.2 \\
4\end{array}$ & $\begin{array}{l}0.29,5.2 \\
9\end{array}$ & $\begin{array}{l}1.3 \\
7\end{array}$ & $\begin{array}{l}0.36,5.3 \\
0\end{array}$ & $\begin{array}{l}2.3 \\
2\end{array}$ & $\begin{array}{l}0.80,6 . \\
74\end{array}$ & $\begin{array}{l}2.5 \\
8\end{array}$ & $\begin{array}{l}0.75,8.8 \\
2\end{array}$ \\
\hline $\begin{array}{l}\text { Unemploy } \\
\text { ed }\end{array}$ & $\begin{array}{l}1.0 \\
1 \\
\end{array}$ & $\begin{array}{l}0.60,1 . \\
71\end{array}$ & $\begin{array}{l}0.9 \\
8 \\
\end{array}$ & $\begin{array}{l}0.61,1 . \\
59\end{array}$ & 1.4 & $\begin{array}{l}0.48,4.1 \\
1 \\
\end{array}$ & $\begin{array}{l}1.3 \\
4 \\
\end{array}$ & $\begin{array}{l}0.43,4.1 \\
4 \\
\end{array}$ & $\begin{array}{l}0.3 \\
9 \\
\end{array}$ & $\begin{array}{l}0.05,2 . \\
91\end{array}$ & - & - \\
\hline \multicolumn{13}{|c|}{ Parental housing conditions } \\
\hline $\begin{array}{l}\text { Low } \\
\text { tenant }\end{array}$ & $\begin{array}{l}1.5 \\
6\end{array}$ & $\begin{array}{l}0.93,2 . \\
60\end{array}$ & 1.4 & $\begin{array}{l}0.82,2 . \\
38\end{array}$ & \begin{tabular}{|l|}
6.2 \\
5 \\
\end{tabular} & $\begin{array}{l}2.24,17 \\
42\end{array}$ & $\begin{array}{l}3.5 \\
4\end{array}$ & $\begin{array}{l}1.09,11 . \\
52\end{array}$ & $\begin{array}{l}1.8 \\
2\end{array}$ & $\begin{array}{l}0.63,5 . \\
27\end{array}$ & $\begin{array}{l}1.0 \\
2\end{array}$ & $\begin{array}{l}0.26,3.9 \\
7\end{array}$ \\
\hline Mid tenant & $\begin{array}{l}1.6 \\
5\end{array}$ & $\begin{array}{l}0.82,3 . \\
34\end{array}$ & $\begin{array}{l}2.1 \\
1 \\
\end{array}$ & $\begin{array}{l}1.14,3 . \\
91\end{array}$ & \begin{tabular}{|l|l|}
1.6 \\
4
\end{tabular} & $\begin{array}{l}0.20,13 . \\
58\end{array}$ & $\begin{array}{l}1.6 \\
6\end{array}$ & $\begin{array}{l}0.24,11 . \\
38\end{array}$ & $\begin{array}{l}0.8 \\
7\end{array}$ & $\begin{array}{l}0.11,6 . \\
74\end{array}$ & - & - \\
\hline $\begin{array}{l}\text { High } \\
\text { tenant }\end{array}$ & $\begin{array}{l}0.8 \\
1\end{array}$ & $\begin{array}{l}0.39,1 . \\
70\end{array}$ & $\begin{array}{l}0.9 \\
6\end{array}$ & $\begin{array}{l}0.52,1 . \\
79\end{array}$ & 2.7 & $\begin{array}{l}0.68,10 . \\
71\end{array}$ & $\begin{array}{l}1.8 \\
2\end{array}$ & $\begin{array}{l}0.43,7.7 \\
3\end{array}$ & $\begin{array}{l}0.9 \\
4\end{array}$ & $\begin{array}{l}0.21,4 . \\
25\end{array}$ & $\begin{array}{l}0.8 \\
7\end{array}$ & $\begin{array}{l}0.18,4.1 \\
5\end{array}$ \\
\hline $\begin{array}{l}\text { Low } \\
\text { owner }\end{array}$ & 1.3 & $\begin{array}{l}0.85,2 . \\
00\end{array}$ & $\begin{array}{l}1.3 \\
7\end{array}$ & $\begin{array}{l}0.92,2 . \\
02\end{array}$ & $\begin{array}{l}1.6 \\
7\end{array}$ & $\begin{array}{l}0.51,5.4 \\
7\end{array}$ & $\begin{array}{l}1.3 \\
5\end{array}$ & $\begin{array}{l}0.42,4.3 \\
6\end{array}$ & $\begin{array}{l}1.2 \\
6\end{array}$ & $\begin{array}{l}0.49,3 . \\
24\end{array}$ & $\begin{array}{l}0.8 \\
3\end{array}$ & $\begin{array}{l}0.27,2.5 \\
9\end{array}$ \\
\hline
\end{tabular}




\begin{tabular}{|c|c|c|c|c|c|c|c|c|c|c|c|c|}
\hline $\begin{array}{l}\text { Mid } \\
\text { owner }\end{array}$ & 0.9 & $\begin{array}{l}0.43,1 . \\
90\end{array}$ & $\begin{array}{l}0.9 \\
3\end{array}$ & $\begin{array}{l}0.49,1 . \\
79\end{array}$ & $\begin{array}{l}3.0 \\
6\end{array}$ & $\begin{array}{l}0.76,12 . \\
27\end{array}$ & $\begin{array}{l}3.1 \\
3\end{array}$ & $\begin{array}{l}0.89,10 . \\
97\end{array}$ & $\begin{array}{l}0.5 \\
6\end{array}$ & $\begin{array}{l}0.07,4 . \\
37\end{array}$ & $\begin{array}{l}0.5 \\
4\end{array}$ & $\begin{array}{l}0.07,4.2 \\
8\end{array}$ \\
\hline $\begin{array}{l}\text { High } \\
\text { owner }\end{array}$ & 1 & - & 1 & - & 1 & - & 1 & - & 1 & - & 1 & - \\
\hline \multicolumn{13}{|c|}{ Parental area deprivation } \\
\hline Q1 & 1 & - & 1 & - & 1 & - & 1 & - & 1 & - & 1 & - \\
\hline Q2 & $\begin{array}{l}1.4 \\
2\end{array}$ & $\begin{array}{l}0.81,2 . \\
51\end{array}$ & $\begin{array}{l}1.1 \\
9\end{array}$ & $\begin{array}{l}0.73,1 . \\
94\end{array}$ & \begin{tabular}{|l|}
1.0 \\
7
\end{tabular} & $\begin{array}{l}0.27,4.2 \\
7\end{array}$ & $\begin{array}{l}0.6 \\
3\end{array}$ & $\begin{array}{l}0.17,2.3 \\
2\end{array}$ & $\begin{array}{l}1.0 \\
7\end{array}$ & $\begin{array}{l}0.35,3 . \\
33\end{array}$ & $\begin{array}{l}1.1 \\
1\end{array}$ & $\begin{array}{l}0.32,3.8 \\
6\end{array}$ \\
\hline Q3 & $\begin{array}{l}1.1 \\
1\end{array}$ & $\begin{array}{l}0.61,2 . \\
04\end{array}$ & $\begin{array}{l}0.9 \\
7\end{array}$ & $\begin{array}{l}0.58,1 . \\
63\end{array}$ & \begin{tabular}{|l|}
1.6 \\
7 \\
\end{tabular} & $\begin{array}{l}0.47,5.9 \\
3\end{array}$ & $\begin{array}{l}1.1 \\
7\end{array}$ & $\begin{array}{l}0.38,3.5 \\
9\end{array}$ & $\begin{array}{l}1.1 \\
2\end{array}$ & $\begin{array}{l}0.36,3 . \\
49\end{array}$ & $\begin{array}{l}0.8 \\
5\end{array}$ & $\begin{array}{l}0.22,3.1 \\
8\end{array}$ \\
\hline Q4 & $\begin{array}{l}1.8 \\
9\end{array}$ & $\begin{array}{l}1.10,3 . \\
26\end{array}$ & $\begin{array}{l}1.5 \\
3\end{array}$ & $\begin{array}{l}0.95,2 . \\
48\end{array}$ & \begin{tabular}{|l|}
2.3 \\
4
\end{tabular} & $\begin{array}{l}0.70,7.7 \\
8\end{array}$ & 0.9 & $\begin{array}{l}0.28,2.9 \\
5\end{array}$ & 0.2 & $\begin{array}{l}0.02,1 . \\
64\end{array}$ & $\begin{array}{l}0.1 \\
8\end{array}$ & $\begin{array}{l}0.02,1.6 \\
2\end{array}$ \\
\hline Q5 & $\begin{array}{l}1.4 \\
3\end{array}$ & $\begin{array}{l}0.81,2 . \\
53\end{array}$ & $\begin{array}{l}1.2 \\
1\end{array}$ & $\begin{array}{l}0.71,2 . \\
06\end{array}$ & \begin{tabular}{l|}
1.1 \\
1 \\
\end{tabular} & $\begin{array}{l}0.28,4.4 \\
4\end{array}$ & $\begin{array}{l}0.2 \\
9\end{array}$ & $\begin{array}{l}0.06,1.3 \\
9\end{array}$ & 1.3 & $\begin{array}{l}0.44,3 . \\
86\end{array}$ & $\begin{array}{l}0.7 \\
6\end{array}$ & $\begin{array}{l}0.18,3.2 \\
2\end{array}$ \\
\hline \multicolumn{13}{|c|}{ Personal education } \\
\hline Low & 1.1 & $\begin{array}{l}0.63,1 . \\
92\end{array}$ & 0.8 & $\begin{array}{l}0.46,1 . \\
39\end{array}$ & \begin{tabular}{|l|}
7.5 \\
3
\end{tabular} & $\begin{array}{l}2.53,22 \\
36\end{array}$ & $\begin{array}{l}4.5 \\
2\end{array}$ & $\begin{array}{l}1.32,15 . \\
46\end{array}$ & 2.9 & $\begin{array}{l}1.03,8 . \\
14\end{array}$ & $\begin{array}{l}3.1 \\
2\end{array}$ & $\begin{array}{l}0.94,10 . \\
31\end{array}$ \\
\hline Mid & $\begin{array}{l}1.2 \\
2\end{array}$ & $\begin{array}{l}0.85,1 . \\
76\end{array}$ & $\begin{array}{l}1.0 \\
7\end{array}$ & $\begin{array}{l}0.76,1 . \\
51\end{array}$ & \begin{tabular}{|l|}
2.8 \\
9
\end{tabular} & $\begin{array}{l}1.00,8.3 \\
1\end{array}$ & $\begin{array}{l}2.9 \\
5\end{array}$ & $\begin{array}{l}1.05,8.3 \\
0\end{array}$ & $\begin{array}{l}1.4 \\
6\end{array}$ & $\begin{array}{l}0.59,3 . \\
58\end{array}$ & $\begin{array}{l}1.1 \\
7\end{array}$ & $\begin{array}{l}0.41,3.3 \\
2\end{array}$ \\
\hline \multirow[t]{3}{*}{ High } & 1 & - & 1 & - & 1 & - & 1 & - & 1 & - & 1 & - \\
\hline & \multicolumn{4}{|c|}{ Brain } & \multicolumn{4}{|c|}{ Non-Hodgkin lymphoma } & \multicolumn{4}{|c|}{ Leukaemia } \\
\hline & \multicolumn{2}{|c|}{\begin{tabular}{|l|} 
Model 1 \\
\end{tabular}} & \multicolumn{2}{|c|}{ Model 2} & \multicolumn{2}{|c|}{ Model 1} & \multicolumn{2}{|c|}{ Model 2} & \multicolumn{2}{|c|}{ Model 1} & \multicolumn{2}{|c|}{ Model 2} \\
\hline \multicolumn{13}{|c|}{ Parental education } \\
\hline Low & $\begin{array}{l}1.1 \\
6\end{array}$ & $\begin{array}{l}0.63,2 . \\
15\end{array}$ & $\begin{array}{l}0.9 \\
2\end{array}$ & $\begin{array}{l}0.42,2 . \\
02\end{array}$ & \begin{tabular}{|l|}
1.7 \\
3
\end{tabular} & $\begin{array}{l}0.63,4.7 \\
4\end{array}$ & 0.9 & $\begin{array}{l}0.29,2.7 \\
3\end{array}$ & $\begin{array}{l}1.4 \\
9\end{array}$ & $\begin{array}{l}0.76,2 . \\
94\end{array}$ & $\begin{array}{l}1.2 \\
2\end{array}$ & $\begin{array}{l}0.53,2.7 \\
9\end{array}$ \\
\hline Mid & $\begin{array}{l}1.1 \\
7\end{array}$ & $\begin{array}{l}0.59,2 . \\
31\end{array}$ & $\begin{array}{l}0.9 \\
2\end{array}$ & $\begin{array}{l}0.42,2 . \\
01\end{array}$ & \begin{tabular}{|l|}
0.5 \\
8
\end{tabular} & $\begin{array}{l}0.14,2.4 \\
4\end{array}$ & $\begin{array}{l}0.5 \\
1\end{array}$ & $\begin{array}{l}0.14,1.8 \\
5\end{array}$ & $\begin{array}{l}1.5 \\
5\end{array}$ & $\begin{array}{l}0.75,3 . \\
19\end{array}$ & $\begin{array}{l}1.3 \\
6\end{array}$ & $\begin{array}{l}0.61,3.0 \\
1\end{array}$ \\
\hline High & 1 & - & 1 & - & 1 & $\begin{array}{l}1.00,1.0 \\
0\end{array}$ & 1 & $\begin{array}{l}1.00,1.0 \\
0\end{array}$ & 1 & $\begin{array}{l}1.00,1 . \\
00\end{array}$ & 1 & $\begin{array}{l}1.00,1.0 \\
0\end{array}$ \\
\hline \multicolumn{13}{|c|}{ Parental occupation } \\
\hline Employed & 1 & - & 1 & - & 1 & $\begin{array}{l}1.00,1.0 \\
0\end{array}$ & 1 & $\begin{array}{l}1.00,1.0 \\
0\end{array}$ & 1 & $\begin{array}{l}1.00,1 . \\
00\end{array}$ & 1 & $\begin{array}{l}1.00,1.0 \\
0\end{array}$ \\
\hline $\begin{array}{l}\text { Looking } \\
\text { for a job }\end{array}$ & $\begin{array}{l}0.7 \\
3 \\
\end{array}$ & $\begin{array}{l}0.23,2 . \\
34\end{array}$ & $\begin{array}{l}0.5 \\
4 \\
\end{array}$ & $\begin{array}{l}0.13,2 . \\
34\end{array}$ & \begin{tabular}{|l|}
1.2 \\
1 \\
\end{tabular} & $\begin{array}{l}0.29,5.1 \\
6\end{array}$ & $\begin{array}{l}1.0 \\
4 \\
\end{array}$ & $\begin{array}{l}0.28,3.8 \\
7 \\
\end{array}$ & 0.7 & $\begin{array}{l}0.22,2 . \\
25\end{array}$ & $\begin{array}{l}0.6 \\
1 \\
\end{array}$ & $\begin{array}{l}0.14,2.6 \\
7\end{array}$ \\
\hline $\begin{array}{l}\text { Unemploy } \\
\text { ed }\end{array}$ & $\begin{array}{l}2.0 \\
8 \\
\end{array}$ & $\begin{array}{l}1.10,3 . \\
91\end{array}$ & $\begin{array}{l}1.5 \\
3 \\
\end{array}$ & $\begin{array}{l}0.68,3 . \\
45\end{array}$ & \begin{tabular}{|l|}
0.8 \\
3 \\
\end{tabular} & $\begin{array}{l}0.19,3.5 \\
7\end{array}$ & $\begin{array}{l}0.4 \\
2 \\
\end{array}$ & $\begin{array}{l}0.07,2.3 \\
7 \\
\end{array}$ & $\begin{array}{l}0.8 \\
5 \\
\end{array}$ & $\begin{array}{l}0.34,2 . \\
14 \\
\end{array}$ & $\begin{array}{l}0.6 \\
5 \\
\end{array}$ & $\begin{array}{l}0.19,2.1 \\
6 \\
\end{array}$ \\
\hline \multicolumn{13}{|c|}{ Parental housing conditions } \\
\hline $\begin{array}{l}\text { Low } \\
\text { tenant }\end{array}$ & $\begin{array}{l}0.8 \\
7\end{array}$ & $\begin{array}{l}0.38,1 . \\
97\end{array}$ & $\begin{array}{l}0.7 \\
1 \\
\end{array}$ & $\begin{array}{l}0.27,1 . \\
89\end{array}$ & \begin{tabular}{|l|}
2.8 \\
4 \\
\end{tabular} & $\begin{array}{l}0.90,8.9 \\
1\end{array}$ & \begin{tabular}{|l|}
4.9 \\
4
\end{tabular} & $\begin{array}{l}1.25,19 . \\
63\end{array}$ & $\begin{array}{l}1.0 \\
2\end{array}$ & $\begin{array}{l}0.47,2 . \\
22\end{array}$ & $\begin{array}{l}0.8 \\
6 \\
\end{array}$ & $\begin{array}{l}0.32,2.2 \\
7\end{array}$ \\
\hline Mid tenant & $\begin{array}{l}0.5 \\
9\end{array}$ & $\begin{array}{l}0.14,2 . \\
48\end{array}$ & $\begin{array}{l}0.5 \\
9\end{array}$ & $\begin{array}{l}0.13,2 . \\
63\end{array}$ & $\begin{array}{l}2.7 \\
2 \\
\end{array}$ & $\begin{array}{l}0.56,13 . \\
09\end{array}$ & $\begin{array}{l}2.9 \\
9\end{array}$ & $\begin{array}{l}0.41,21 . \\
52\end{array}$ & $\begin{array}{l}0.6 \\
1\end{array}$ & $\begin{array}{l}0.15,2 . \\
55\end{array}$ & $\begin{array}{l}0.3 \\
5\end{array}$ & $\begin{array}{l}0.05,2.6 \\
7\end{array}$ \\
\hline $\begin{array}{l}\text { High } \\
\text { tenant }\end{array}$ & 0.8 & $\begin{array}{l}0.31,2 . \\
05\end{array}$ & $\begin{array}{l}0.8 \\
5\end{array}$ & $\begin{array}{l}0.32,2 . \\
27\end{array}$ & \begin{tabular}{|l|}
0.7 \\
3 \\
\end{tabular} & $\begin{array}{l}0.09,5.9 \\
8\end{array}$ & $\begin{array}{l}1.6 \\
1\end{array}$ & $\begin{array}{l}0.24,10 . \\
97\end{array}$ & $\begin{array}{l}0.4 \\
9\end{array}$ & $\begin{array}{l}0.15,1 . \\
61\end{array}$ & $\begin{array}{l}0.5 \\
3 \\
\end{array}$ & $\begin{array}{l}0.16,1.7 \\
7\end{array}$ \\
\hline $\begin{array}{l}\text { Low } \\
\text { owner }\end{array}$ & $\begin{array}{l}0.9 \\
2 \\
\end{array}$ & $\begin{array}{l}0.50,1 . \\
70\end{array}$ & $\begin{array}{l}0.7 \\
1 \\
\end{array}$ & $\begin{array}{l}0.34,1 . \\
50\end{array}$ & $\begin{array}{l}2.2 \\
4 \\
\end{array}$ & $\begin{array}{l}0.81,6.2 \\
1\end{array}$ & $\begin{array}{l}3.7 \\
4\end{array}$ & $\begin{array}{l}1.11,12 . \\
60\end{array}$ & $\begin{array}{l}1.0 \\
1 \\
\end{array}$ & $\begin{array}{l}0.55,1 . \\
85\end{array}$ & $\begin{array}{l}0.8 \\
3 \\
\end{array}$ & $\begin{array}{l}0.41,1.7 \\
0\end{array}$ \\
\hline $\begin{array}{l}\text { Mid } \\
\text { owner }\end{array}$ & $\begin{array}{l}1.1 \\
7 \\
\end{array}$ & $\begin{array}{l}0.49,2 . \\
79\end{array}$ & $\begin{array}{l}0.8 \\
6 \\
\end{array}$ & $\begin{array}{l}0.30,2 . \\
50\end{array}$ & \begin{tabular}{|l|}
2.6 \\
7 \\
\end{tabular} & $\begin{array}{l}0.69,10 . \\
31\end{array}$ & $\begin{array}{l}6.2 \\
5 \\
\end{array}$ & $\begin{array}{l}1.61,24 . \\
30\end{array}$ & 0.2 & $\begin{array}{l}0.03,1 . \\
47\end{array}$ & $\begin{array}{l}0.2 \\
2 \\
\end{array}$ & $\begin{array}{l}0.03,1.6 \\
4 \\
\end{array}$ \\
\hline $\begin{array}{l}\text { High } \\
\text { owner }\end{array}$ & 1 & - & 1 & - & 1 & - & 1 & - & 1 & - & 1 & - \\
\hline
\end{tabular}




\begin{tabular}{|c|c|c|c|c|c|c|c|c|c|c|c|c|}
\hline \multicolumn{13}{|c|}{ Parental area deprivation } \\
\hline Q1 & 1 & - & 1 & - & 1 & - & 1 & - & 1 & - & 1 & - \\
\hline Q2 & $\begin{array}{l}0.3 \\
2\end{array}$ & $\begin{array}{l}0.12,0 . \\
86\end{array}$ & $\begin{array}{l}0.2 \\
8\end{array}$ & $\begin{array}{l}0.09,0 . \\
84\end{array}$ & $\begin{array}{l}1.3 \\
4\end{array}$ & $\begin{array}{l}0.36,5.0 \\
1\end{array}$ & $\begin{array}{l}1.8 \\
8\end{array}$ & $\begin{array}{l}0.45,7.9 \\
0\end{array}$ & $\begin{array}{l}0.9 \\
3\end{array}$ & $\begin{array}{l}0.44,1 . \\
96\end{array}$ & $\begin{array}{l}0.9 \\
3\end{array}$ & $\begin{array}{l}0.42,2.0 \\
7\end{array}$ \\
\hline Q3 & $\begin{array}{l}1.0 \\
6\end{array}$ & $\begin{array}{l}0.54,2 . \\
10\end{array}$ & $\begin{array}{l}0.9 \\
4\end{array}$ & $\begin{array}{l}0.44,2 . \\
03\end{array}$ & $\begin{array}{l}0.8 \\
4\end{array}$ & $\begin{array}{l}0.19,3.7 \\
8\end{array}$ & $\begin{array}{l}0.9 \\
3\end{array}$ & $\begin{array}{l}0.18,4.8 \\
8\end{array}$ & 0.9 & $\begin{array}{l}0.42,1 . \\
93\end{array}$ & 0.8 & $\begin{array}{l}0.34,1.8 \\
8\end{array}$ \\
\hline Q4 & $\begin{array}{l}0.7 \\
7\end{array}$ & $\begin{array}{l}0.36,1 . \\
64\end{array}$ & $\begin{array}{l}0.7 \\
1\end{array}$ & $\begin{array}{l}0.30,1 . \\
68\end{array}$ & $\begin{array}{l}1.4 \\
8\end{array}$ & $\begin{array}{l}0.40,5.5 \\
3\end{array}$ & $\begin{array}{l}2.2 \\
8\end{array}$ & $\begin{array}{l}0.55,9.4 \\
1\end{array}$ & $\begin{array}{l}0.7 \\
9\end{array}$ & $\begin{array}{l}0.36,1 . \\
77\end{array}$ & $\begin{array}{l}0.7 \\
5\end{array}$ & $\begin{array}{l}0.30,1.8 \\
3\end{array}$ \\
\hline Q5 & $\begin{array}{l}0.9 \\
8 \\
\end{array}$ & $\begin{array}{l}0.49,1 . \\
96\end{array}$ & \begin{tabular}{|l|}
1.0 \\
5 \\
\end{tabular} & $\begin{array}{l}0.45,2 . \\
42\end{array}$ & $\begin{array}{l}1.9 \\
4 \\
\end{array}$ & $\begin{array}{l}0.57,6.6 \\
4 \\
\end{array}$ & $\begin{array}{l}2.1 \\
1 \\
\end{array}$ & $\begin{array}{l}0.49,9.1 \\
4 \\
\end{array}$ & $\begin{array}{l}0.7 \\
4 \\
\end{array}$ & $\begin{array}{l}0.33,1 . \\
65 \\
\end{array}$ & $\begin{array}{l}0.5 \\
2 \\
\end{array}$ & $\begin{array}{l}0.18,1.4 \\
8\end{array}$ \\
\hline \multicolumn{13}{|c|}{ Personal education } \\
\hline Low & $\begin{array}{l}2.0 \\
8\end{array}$ & $\begin{array}{l}0.94,4 . \\
62\end{array}$ & $\begin{array}{l}2.2 \\
8\end{array}$ & $\begin{array}{l}0.91,5 . \\
67\end{array}$ & $\begin{array}{l}0.9 \\
7\end{array}$ & $\begin{array}{l}0.21,4.4 \\
7\end{array}$ & $\begin{array}{l}0.8 \\
1\end{array}$ & $\begin{array}{l}0.20,3.3 \\
2\end{array}$ & 2.3 & $\begin{array}{l}1.15,4 . \\
63\end{array}$ & $\begin{array}{l}3.9 \\
4\end{array}$ & $\begin{array}{l}1.76,8.8 \\
3\end{array}$ \\
\hline Mid & 2.2 & $\begin{array}{l}1.25,3 . \\
88\end{array}$ & $\begin{array}{l}2.2 \\
6 \\
\end{array}$ & $\begin{array}{l}1.19,4 . \\
29\end{array}$ & $\begin{array}{l}1.7 \\
5 \\
\end{array}$ & $\begin{array}{l}0.74,4.1 \\
5\end{array}$ & $\begin{array}{l}1.2 \\
8 \\
\end{array}$ & $\begin{array}{l}0.52,3.1 \\
5\end{array}$ & 1.3 & $\begin{array}{l}0.73,2 . \\
32\end{array}$ & $\begin{array}{l}1.4 \\
8 \\
\end{array}$ & $\begin{array}{l}0.75,2.9 \\
2\end{array}$ \\
\hline High & 1 & - & 1 & - & 1 & - & 1 & - & 1 & - & 1 & - \\
\hline
\end{tabular}

*Model 1: adjusted for current age; Model 2: adjusted for current age, parental and personal SEP.

Table 5: Relative site-specific cancer mortality inequalities (MRRs) by parental and personal SEP among Belgian female young adults, 2001-2011.

\section{Discussion}

\subsection{Methodological issues}

This study uses nationwide administrative data allowing for precise estimates of overall and site-specific cancer mortality among young adults at the individual level. To account for gender differences in the social patterning of young-adult cancer mortality, analyses were conducted separately for men and women, $[9,15,20]$. Yet, we need to bear in mind that the number of site-specific cancer deaths are rather low.

The nature of the data avoids recall bias concerning childhood SE circumstances [16]. Consequently, this study adds to the literature by including indicators of both parental and personal SEP to explain young adult cancer mortality, as was recommended by previous research [21]. Moreover, while most studies usually assess one SEP indicator (often father's occupation), we included multiple measures of parental SEP: education, occupational status and housing conditions, as well as area deprivation of the municipality of residence. Indicators of SEP are not interchangeable but tap into other causal processes, and therefore multiple indicators should be considered simultaneously [20]. By doing this, we were able to identify the net effects of each indicator [20], as well as the relative importance of these indicators in explaining young adult site-specific cancer mortality [7]. Moreover, we were able to account for the educational level of the young adults. Instead of using highest obtained educational level, we decided to use current educational level, which has shown to be an appropriate indicator of future SEP of young adults [16]. Even though they would not finalize the education they are currently following, the influence of peers during this period may be a determinant of their health [15]. Using completed education would have implied an underestimation of education because one out of four was still enrolled in education at the census. However, using current educational level may at the same time imply an overestimation of educational attainment. Complete-case analyses have been conducted. 
We also performed some additional sensitivity analyses including the missing values as separate categories, leading to the conclusion that the observed results are even an underestimation of social inequalities in young adult mortality.

To account for a health selection effect regarding the association between personal education and cancer mortality, we performed some sensitivity analyses including SRH of the young adults, measured at the census of 2001. These results suggest that this is an important issue for some cancer sites. For example, low-educated men and women were more likely to die from leukaemia, but this association was no longer significant after adjustment for SRH. The observed relation between personal education and mortality from leukaemia might be explained by the fact that these persons were already too sick to attain a higher level of education.

A limitation of our dataset is the lack of information on incidence or survival. Distinguishing between incidence, survival and mortality is important in identifying the causal pathways linking SEP and cancer mortality [3, 10]. Higher SEP can be a risk factor for the onset of disease, but can at the same time be a protective factor for survival, as in the case of breast cancer for example [10]. In this way, effects of SEP might counteract and, hence, be hidden when taking only mortality as an outcome. Moreover, due to the lack of data on behavioural risk factors and health care use, it is difficult to explain the observed SE inequalities in mortality [3, 15, 22, 23].

\subsection{Theoretical issues}

Our results revealed a strong association between both parental and personal SEP and overall cancer mortality. This is not in line with other studies that did not observe an association between childhood SEP and overall cancer mortality [3,7]. The overall tendency in literature is that other COD such as cardiovascular diseases are more related to cumulative disadvantage, whereas cancer is more strongly associated with adult SEP [4]. In our study, personal SEP also seemed to be the strongest predictor in the fully adjusted models, although for women this was partly due to a health selection effect. After adjustment for adult SEP, parental education was no longer associated with cancer mortality but housing conditions and living in deprived areas still was. However, parental SEP probably still has an indirect effect on health through its effect on personal education [16].

Social inequalities in site-specific cancer mortality seemed more expressed among women than among men. This might be due to the fact that within this age group, men are more likely to die from external causes (accidents, suicide and injuries) $[8,15]$. Hence, men in low SEP groups might show more risky behaviours that might increase their risk for other diseases such as injuries [15]. In women, personal education seemed to be the most important predictor of young-adult cancer mortality, even after adjusting for the other SEP indicators. Educational inequalities were observed in cancers of the cervix, ovary, brain, melanoma and leukaemia. Yet, in the cases of ovarian cancer, brain cancer and leukaemia, the effects were explained after adjustment for SRH. In men, a similar pattern was observed for leukaemia. This could point to a health selection effect: these individuals did not attain a high personal educational level in young adult because of health problems during childhood. Next to personal SEP, cervical cancer mortality was also related to parental SEP, which is in line with previous research [9]. This association might be due 
to a higher prevalence of HPV infection due to limited access to health care to detect and treat pre-cancerous lesions $[24,25]$. Breast cancer appears to be higher in women who grew up in disadvantaged living conditions, both in terms of housing conditions and area deprivation. One study also observed higher breast cancer mortality rates in low childhood SEP groups [3], whereas others did not observe a strong association [7]. In our study, personal SEP was not associated with breast cancer mortality, while literature suggests higher breast cancer mortality among higheducated women due to reproductive factors, such as later age at first pregnancy [3]. We did not observe such an association, probably because of the relatively young age of our study population. In men, both parental and personal education were associated with colorectal cancer. However, the associations were no longer significant by adding al variables into one model. The same association with parental education was found in other studies [3,5], and might be explained by differences in dietary practices [3].

Moreover, poor housing conditions resulted in higher male lung cancer mortality. Some studies also observed lung cancer mortality to be related to childhood SEP [9, 14, 26]. A study of Dedman and colleagues [26] showed higher lung cancer mortality in people that grew up in poor housing conditions, independently of adult SEP. Especially poor ventilation resulted in higher lung cancer mortality, which may reflect an increased exposure to tobacco smoke, fume from cookers and fires or other types of pollutants. However, the general trend in literature is that lung cancer mortality is more related to personal than to parental SEP due to differences in smoking behaviour [1, 3, 5-7]. This was not the case in our study, probably due to the relatively young age of the study population. Finally, a surprising finding was that low-educated women had a three-fold higher melanoma mortality compared with high-educated women in the fully adjusted model. This is not in line with literature which shows either no association [3] or an inverse association with adult SEP [5].

Our results stress the importance of including different indicators, which tap into different causal pathways when studying the social patterning of cause-specific mortality [4, 27]. Education captures the human capital, is related to the ability to act upon health education messages, and serves as a measure of prestige at young adulthood [28, 29]. Housing conditions are related to material and financial resources, and hence to health care utilization, as well as to the likelihood of making healthy choices, e.g. in terms of buying healthy food [20,30]. Moreover, it can have a direct effect on health through the quality of the housing, for example through the adequacy of the ventilation of the dwelling [26]. Occupational status on the other hand reflects the ability to realize this human capital into the labour market, and is likely to capture material resources, access to health care, social networks as well as occupational exposures [27]. Not only parental social class but also living in a deprived area was associated with higher mortality, in line with a previous study [4]. Area deprivation is related to health through aspects of both the physical environment (e.g. physical activity resources in recreational settings or local healthy food facilities) and the social environment (e.g. social norms and stressors, or social cohesion) [31].

Consistent with literature [4], personal education seemed to be a very strong indicator of young-adult cancer mortality, independent of parental SEP. Overall cancer mortality was higher in low-educated young adults, which can be explained by three mechanisms [32]. The first one is health selection which implies that adults are low- 
educated because of their bad health. The sensitivity analyses adjusting for SRH proved that this was the case for overall cancer mortality in low-educated women, and for educational differences in leukaemia. The second explanation is indirect selection: the social circumstances early in life determine one's personal SEP in later life and hence one's health [32]. However, our data show, that the association between personal SEP and cancer mortality is independent of parental SEP. Therefore, the third mechanism of social causation is more likely to explain the association between cancer mortality and personal education. Social causation considers health as a direct result of education itself [7, 32]. Different aspects associated with low SEP (e.g. lack of social support or risk-taking behaviour), might then explain these differential mortality patterns.

There was quite some variation in site-specific cancer mortality patterns, probably due to the different aetiological processes behind these cancers [14]. Inequalities in cancer mortality were more pronounced for personal education and living circumstances during childhood (i.e. housing conditions and area deprivation). Subjects living in worse quality housing may experience increased and cumulative exposures to pollutants such as fumes or smoke [3, 26]. However, the associations with parental education were attenuated when personal education was added to the model. This points to the fact that inequalities in young adult cancer mortality are mainly related to the social patterning of health behaviours (i.e. smoking) during adulthood $[1,3,5,6,8,15,33]$, and probably also to an indirect effect of parental SEP through personal SEP [32]. Yet, Hart and colleagues [11] showed that the effects of adulthood social class on cancer mortality persisted after adjustment for risk factors, including smoking whereas Power and colleagues [8] showed that adjustment for adult SEP and smoking and BMI eliminated inequalities in lung cancer mortality.

\section{Conclusion}

Cancer mortality during young adulthood is not only related to SEP during the short period before death, but also to the SE circumstances during childhood. Health inequalities associated with adverse childhood conditions are thus not only continuities in SEP but also long lasting exposures from childhood [8]. This shows the importance of adopting a life-course perspective when studying the social distribution of health and mortality $[1,4,6,8,9]$. Focussing on one point in time (whether childhood or adulthood) might not be sufficient to unravel the social patterning of health inequalities [1]. Hence, the same is true for policy: measures against social inequalities in health should focus on all stages throughout the life course $[9,21]$. Children who experience strong social support and who are given the opportunity of taking healthy options, will be able to develop cognitive skills that will lead to healthier behaviours, which will decrease their disease risks [3]. Consequently, policy measures to improve development in early life will in turn lead to health-enhancing behaviours in adulthood. This, in combination with policies that stimulate making healthy choices in adulthood (e.g. increase of price of unhealthy products), as well as individuallevel interventions (e.g. smoking cessation services) are important to reduce health inequalities [3].

To summarize, the health effects of adverse early SE circumstances can be reduced or even eliminated by personal education and its related resources in adulthood [16]. This points to the potential benefits that education can have for 
one's health by improving one's SEP [34]. Therefore, improving the current (and future) social conditions of children might be reflected in decreasing health inequalities within some decades [6].

\section{References}

1. Claussen B, Smith GD, Thelle DS. Impact of childhood and adulthood socioeconomic position on cause specific mortality: the Oslo Mortality Study. Journal of Epidemiology and Community Health 57 (2003): 40-45.

2. Tani Y, Kondo N, Nagamine Y, et al. Childhood socioeconomic disadvantage is associated with lower mortality in older Japanese men: the JAGES cohort study. International Journal of Epidemiology (2016): 90.

3. Vohra J, Marmot MG, Bauld L, et al. Socioeconomic position in childhood and cancer in adulthood : a rapid-review. Journal of Epidemiology \& Community Health 70 (2016): 629-634.

4. Davey Smith G, Hart C, Blane D, et al. Lifetime socioeconomic position and mortality: prospective observational study. BMJ 314 (1997): 547-552.

5. Næss Ø, Claussen B, Smith GD. Relative impact of childhood and adulthood socioeconomic conditions on cause specific mortality in men. Journal of Epidemiology and Community Health 58 (2004): 597-598.

6. Davey Smith G, Hart C, Blane D, et al. Adverse socioeconomic conditions in childhood and cause specific adult mortality : prospective observational study. BMJ 316 (1998): 1631-1635.

7. Strand BH, Kunst A. Childhood Socioeconomic Position and Cause-specific Mortality in Early Adulthood. American Journal of Epidemiology 165 (2007): 85-93.

8. Power C, Hyppönen E, Davey Smith G. Socioeconomic Position in Childhood and Early Adult Life and Risk of Mortality : A Prospective Study of the Mothers of the 1958 British Birth Cohort. American Journal of Public Health 95 (2005): 1396-1402.

9. Næss $\varnothing$, Strand BH, Smith GD. Childhood and adulthood socioeconomic position across 20 causes of death: a prospective cohort study of 800000 Norwegian men and women. Journal of Epidemiology and Community Health 61 (2007): 1004-1009.

10. Pudrovska T, Anikputa B. The Role of Early-Life Socioeconomic Status in Breast Cancer Incidence and Mortality: Unraveling Life Course Mechanisms. J Ageing Health 24 (2012): 323-344.

11. Hart CL, Davey Smith G, Blane D. Social mobility and 21year mortality in a cohort of Scottish men. Social Science and Medicine 47 (1998): 1121-1130.

12. Ben-shlomo Y, Kuh D. A life course approach to chronic disease epidemiology: conceptual models, empirical challenges and interdisciplinary perspectives. International Journal of Epidemiology 31 (2002): 285-293.

13. Kuh D, Ben-Shlomo Y, Lynch J, et al. Life course epidemiology. Journal of Epidemiology and Community Health 57 (2003): 778-783.

14. Galobardes B, Lynch JW, Smith GD. Childhood Socioeconomic Circumstances and Cause-specific Mortality in Adulthood: Systematic Review and Interpretation. Epidemiologic Reviews 26 (2004): 7-21. 
15. Lawlor DA, Sterne JAC, Tynelius P, et al. Association of Childhood Socioeconomic Position with Causespecific Mortality in a Prospective Record Linkage Study of 1,839,384 Individuals. American Journal of Epidemiology 164 (2006): 907-915.

16. De Grande H, Vandenheede H, Deboosere P. Educational inequalities in the transition to adulthood in Belgium: The impact of intergenerational mobility on young-adult mortality in 2001-2009. PLoS ONE 10 (2015): 1-18.

17. Deboosere P, Willaert D. Codebook Algemene Socio-economische enquête 2001 (Working Paper 2004-1). Brussel (2004).

18. Deboosere P, Demarest S, Lorant V, et al. Gezondheid en mantelzorg. Monografieën van de SociaalEconomische enquête 2001 (nr. 1). Brussel (2006).

19. Hagedoorn P, Vandenheede H, Vanthomme K, et al. A cohort study into head and neck cancer mortality in Belgium (2001-11): Are individual socioeconomic differences conditional on area deprivation? Oral Oncology 61 (2016): 76-82.

20. Zarnowiecki D, Ball K, Parletta N, et al. Describing socioeconomic gradients in children's diets - does the socioeconomic indicator used matter? The international journal of behavioral nutrition and physical activity 11 (2014): 44.

21. Næss $\varnothing$, Claussen B, Thelle DS, et al. Cumulative Deprivation and Cause Specific Mortality. A Census Based Study of Life Course Influences over three Decades. Journal of Epidemiology and Community Health 58 (2004): 599-603.

22. Saurel-Cubizolles MJ, Chastang JF, Menvielle G, et al. Social inequalities in mortality by cause among men and women in France. Journal of Epidemiology and Community Health 63 (2009): 197-202.

23. Steenland K, Hu S, Walker J. All-cause and cause-specific mortality by socioeconomic status among employed persons in 27 US states, 1984-1997. American Journal of Public Health 94 (2004): 1037-1042.

24. Spadea T, Zengarini N, Kunst A, et al. Cancer risk in relationship to different indicators of adult socioeconomic position in Turin, Italy. Cancer Causes and Control 21 (2010): 1117-1130.

25. de Vries E, Arroyave I, Pardo C, et al. Trends in inequalities in premature cancer mortality by educational level in Colombia, 1998-2007. Journal of epidemiology and community health (2014): 1-8.

26. Dedman DJ, Gunnell D, Smith GD, et al. Childhood housing conditions and later mortality in the Boyd Orr cohort. Journal of Epidemiology and Community Health 55 (2001): 10-15.

27. NÆss $\emptyset$, Claussen B, Thelle DS, et al. Four indicators of socioeconomic position : relative ranking across causes of death. Scandinavian Journal of Public Health 33 (2005): 215-221.

28. Toch-Marquardt M, Menvielle G, Eikemo TA, et al. Occupational class inequalities in all-cause and causespecific mortality among middle-aged men in 14 European populations during the early 2000s. PLoS ONE 9 (2014).

29. Galobardes B, Shaw M, Lawlor DA, et al. Indicators of socioeconomic position (part 1). Journal of Epidemiology and Community Health 60 (2006): 7-12.

30. Merletti F, Galassi C, Spadea T. The socioeconomic determinants of cancer. Environmental health: a global access science source 10 Suppl 1(Suppl 1) (2011): S7. 
31. Diez Roux AV, Mair C. Neighborhoods and health. Ann. N.Y. Acad. Sci 1186 (2010): 125-145.

32. Marmot M, Ryff CD, Bumpass LL, et al. Social inequalities in health: Next questions and converging evidence. Social Science and Medicine 44 (1997): 901-910.

33. Melchior M, Berkman L, Kawachi I, et al. Lifelong socioeconomic trajectory and premature mortality (3565 years) in France: findings from the GAZEL Cohort Study. Journal of Epidemiology and Community Health 60 (2006): 937-944.

34. Heslop P, Davey G, Macleod J, et al. The socioeconomic position of employed women, risk factors and mortality. Social Science and Medicine 53 (2001): 477-485.

Citation: Katrien Vanthomme, Hadewijch Vandenheede, Paulien Hagedoorn, Sylvie Gadeyne. Parental and/or Personal Socioeconomic Circumstances: How to Explain Site-Specific Cancer Mortality Inequalities Among Young Belgian Adults. Journal of Cancer Science and Clinical Therapeutics 2 (2018): 54-72. 